\title{
Provenance of Albian to Cenomanian exotics-bearing turbidites in the Western Carpathians: a heavy mineral analysis
}

\author{
Roman AUBRECHT ${ }^{1,2, *}$, Simona BELLOVÁ ${ }^{1}$ and Tomáš MIKUŠ ${ }^{3}$ \\ 1 Comenius University, Department of Geology and Paleontology, Faculty of Natural Sciences, Ilkovičova 6, 84215 \\ Bratislava, Slovakia \\ 2 Slovak Academy of Sciences, Institute of Earth Sciences - Geophysical Division, Dúbravská cesta 9, 84528 Bratislava, \\ Slovakia \\ 3 Slovak Academy of Sciences, Institute of Earth Sciences, Workplace Banská Bystrica, Ďumbierska 1, SK-974 01 Banská \\ Bystrica, Slovakia
}

Aubrecht, R., Bellová, S., Mikuš, T., 2020. Provenance of Albian to Cenomanian exotics-bearing turbidites in the Western Carpathians: a heavy mineral analysis. Geological Quarterly, 64 (3): 658-680, doi: 10.7306/gq.1543

Throughout the Cretaceous, Tethyan oceanic branches gradually closed, and various ophiolites became obducted and eroded. Their remnants, however, provide an abundance of exotic clasts of unknown origin. Sandstone samples from the oldest, Albian exotics-bearing strata of the Pieniny Klippen Belt and Central Western Carpathians were analysed for heavy minerals. These samples were dominated by a high content of chrome-spinels, zircon, tourmaline, apatite and rutile. Titanite, kyanite, monazite, epidote, sillimanite and staurolite were much less abundant. Garnet was generally also rare; however, it was locally common, as were blue amphiboles, pyroxenes and kyanite. The spinels found in the samples were predominantly derived from harzburgites (supra-subduction peridotites and volcanic rocks). The blue amphiboles represented glaucophanes to ferroglaucophanes, and were derived from HP/LT metabasites. Pyroxenes (enstatite, less commonly augite and diopside) most likely came from coeval volcanics. Most of the tourmalines were derived from metasedimentary rocks and locally from granitoids. Furthermore, some have a complex zonation with two phases of tourmaline, or tourmaline intergrown with quartz. These were likely derived from ophiolitic sources. The results from our analysis indicate a dominance of ophiolites and older sediments with local input of continental crust metamorphic rocks. A resulting palaeogeographic reconstruction involves secondary doubling of the Neotethys suture zone and its lateral shift north of the Central Western Carpathians, which formed a common source for exotics in the Pieniny Klippen Belt and the Central Western Carpathians.

Key words: ophiolites, Cr-spinel, blue amphibole, tourmaline, pyroxenes, Cretaceous palaeogeography.

\section{INTRODUCTION}

The end of the Early Cretaceous was a time of fundamental change across the Tethyan realm. During this time, carbonate sedimentation ceased and was replaced by siliciclastic deposition as a result of gradual closure of various oceanic branches (e.g., Neotethyan, Alpine Atlantic), crustal shortening, formation of accretionary wedges and obduction of ophiolitic complexes. Most of the source complexes were later underthrust, buried or removed by erosion. The only remaining witness of the palaeotectonic and palaeogeographic processes from this period is the detritus of sedimentary rocks. The resulting strata are mostly deep-water clastic deposits containing material of an unknown, exotic origin. In the Western Carpathians, the earliest occurrences of exotic ophiolitic detritus (Cr-spinels) were inter-

\footnotetext{
* Corresponding author, e-mail: roman.aubrecht@uniba.sk
}

Received: February 17, 2020; accepted: April 11, 2020; first published online: July 21,2020 calations of Hauterivian sandstone turbidites in the Fatric and Hronic units (Jablonský, 1992; Jablonský et al., 2001). Cr-spinels had been previously discovered in Aptian pebbles among the Albian exotic detritus (Mišík et al., 1980; Wagreich et al., 1995). The first massive input of exotic material took place during the Albian, and these deposits thus became the subject of thorough pebble analyses (see references below); however, systematic analysis of the sand fraction has so far been lacking. Despite prolonged research, the provenance of the exotic deposits remains mysterious, and their investigation has presented an enormous challenge to date.

The Albian exotics-bearing units (Fig. 1) occur in the Pieniny Klippen Belt (Uhry and Upohlav formations of the Klape Unit), as well as in the Central Western Carpathians (the Poruba Formation within the Tatric and Fatric units). The relatively younger Cenomanian Praznov Formation is located in the Manín Unit. In the Pieniny Klippen Belt, which is a mélange zone consisting of mixed blocks of various units (see the review of Plašienka, 2018), the Klape and Manín units belong among the non-Oravic units of the Pieniny Klippen Belt (typically called the Peri-Klippen Zone; Mahel', 1980). The Klape Unit is of unknown provenance and has been the subject of much debate (e.g., Plašienka, 1995, 1996; Mišík, 1996; see the Discussion sec- 


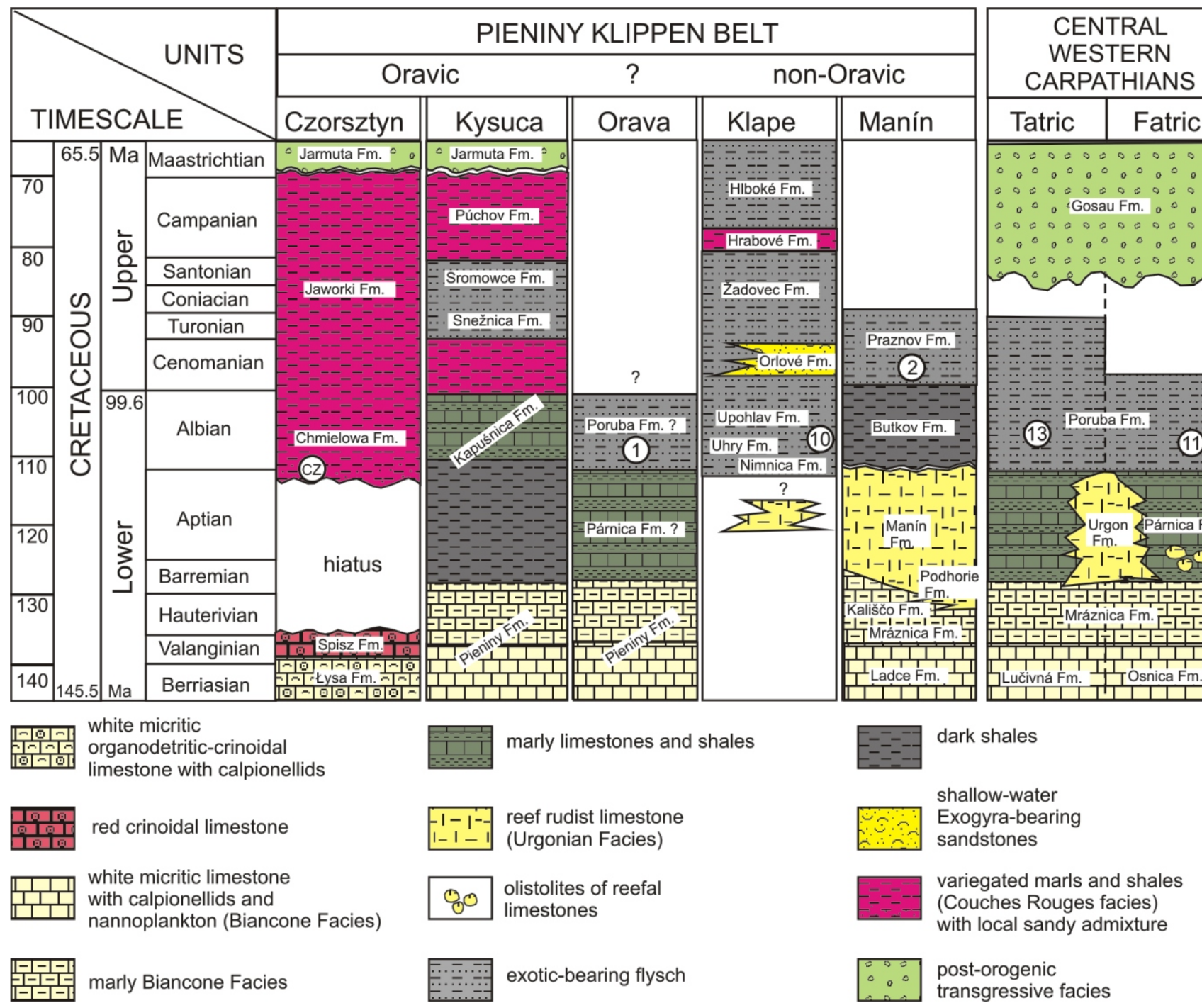

Fig. 1. Schematic lithostratigraphic columns of the exotics-bearing units in the Western Carpathians arranged approximately according to their present positions from north to south (left to right)

The scheme is based on: Birkenmajer (1977), Aubrecht et al. (2009) and Mello et al. (2011). The numbers in the circles represent the numbers of samples analysed from the individual formations; CZ - exotics-bearing sandy admixture in the Upper Aptian/Lower Albian deposits of the Czorsztyn Unit analysed by Aubrecht et al. (2009)

tion). The Manín Unit, which is presently situated in the Pieniny Klippen Belt, is considered to have originally derived from the Central Western Carpathians. There is ongoing discussion as to whether this unit belonged to the Tatric or the Fatric domain (Andrusov, 1938; Mahel', 1978; see also Rakús and Hók, 2005 and the references therein). In the Pieniny Klippen Belt sensu stricto (Oravic units), the input of exotic material began much later, during the Coniacian, being represented by the Sromowce Formation (Birkenmajer, 1977) occurring in deeper-water units of this domain. Albian terrigenous sandy to gravelly input in the Oravic units was scarce, and was represented by only a single occurrence of the Albian flysch (Trawne Member) attributed to the Kysuca Unit (called the Branisko Unit in Poland) as described by Birkenmajer (1987). The most surprising and unexpected finding was the fact that fine exotic ophiolitic material was also registered in Upper Aptian-Albian deposits of the Czorsztyn Unit (Aubrecht et al., 2009). This unit was originally the shallowest of all the Oravic units and thought to be isolated from the exotic clastic input.
There is another occurrence of Albian flysch of unknown attribution in the Pieniny Klippen Belt. It occurs below a large klippe of the Orava Unit (Havranský vrch Hill and Kozinský vrch Hill) near the village of Zázrivá in the region of Orava. Haško (1977) interpreted the superposition as a tectonic contact between the main klippe and Albian turbidites. This was because the Orava Unit is usually positioned toward the Oravic domain, whereas Albian sandy or conglomeratic strata are almost completely absent. However, the attribution of the Orava Unit to the Oravic domain was put into doubt by Mahel' (1986), who inferred that it may have been of more southern Fatric provenance. Our closer inspection of this occurrence during the sampling shows that there is a gradual, continuous transition from the Barremian-Aptian marlstones to the Albian flysch, and the contact seems to be stratigraphic rather than tectonic. Thus, the flysch can be most likely attributed to the Orava Unit.

In the Central Western Carpathians, the Albian exoticsbearing flysch deposition preceded the main, Mediterranean orogenic phase, and lasted until the Middle Turonian, when the 
nappe thrusting took place. In the Klape and Oravic units, sedimentation of exotic material lasted until the end of the Cretaceous. This still consisted of purely exotic detritus with no material derived from the neighbouring Oravic or Central Carpathian units. After the Maastrichtian Laramian collision between these blocks, the exotic deposition continued (Paleogene Jarmuta-Proč formations) mixed with non-exotic material from the neighbouring emerged units. Exotic sources influenced deposition in these formations until the Eocene (Mišík et al., 1991; Winkler and Ślączka, 1992, 1994; Oszczypko and Salata, 2005; Bónová et al., 2017, 2018; Madzin et al., 2019).

The research presented in this paper deals with the Albian-Cenomanian flysch in the Pieniny Klippen Belt and the Central Western Carpathians. The deposits of the Klape Unit were sedimentologically analysed by Marschalko (1986), and the Poruba Formation in the Central Western Carpathians was defined and systematically sedimentologically analysed by Jablonský $(1978,1986)$. The exotic conglomerates of the Klape and Manín units were analysed mostly for their content of carbonate pebbles (Mišík and Sýkora, 1981; Mišík and Marschalko, 1988; Birkenmajer et al., 1990). Pebble analyses of crystalline metamorphic, magmatic and siliciclastic rocks were provided by Krivý (1969), Kamenický et al. (1974), Kamenický and Král' (1979), Šímová (1982, 1985a, b, c), Šímová and Šamajová (1982), Ivan and Sýkora (1993), Uher and Marschalko (1993), Ivan et al. (2006) and Zat'ko and Sýkora (2006). Systematic pebble analysis of carbonate components of the Poruba Formation in the Central Western Carpathians was performed by Mišík et al. (1981).

The abundance of pebble-analysis data caused relative neglect of the sand fraction in analysing the Western Carpathian exotic material; the results included only percentage ratios of the heavy minerals (Mišík et al., 1980; Jablonský, 1986). Percentage ratios from several localities that were accompanied by provenance analysis of spinels were published by Jablonský et al. (2001). Other provenance analyses of the individual minerals included only point locations (e.g., Sýkora et al., 1997; Straka, 2011).

This paper focuses on a systematic provenance analysis of heavy minerals in the Albian-Cenomanian exotics-bearing deposits in the Western Carpathians. Some early preliminary results of this research were published by Bellová et al. (2018) to which the readers are referred for some documentation, e.g. the tables of representative analyses of minerals. Our summary article is complemented by ten new localities, new analyses of heavy minerals, and by petrographic analysis of the sandstones examined. The results are discussed in the context of data from Cretaceous exotics-bearing deposits in other areas of the Tethyan realm.

\section{SAMPLED SITES, MATERIAL AND METHODS}

Samples from 37 localities were analysed for heavy minerals: 10 from the Klape Unit (Uhry and Upohlav formations) and 24 from the Poruba Formation. From the latter, 12 localities from the Tatric units (Albian-Cenomanian) and 12 from the Fatric units (Albian-Turonian) were sampled (Figs. 1 and 2). Additionally, two samples from the Manín Unit and one from the Orava Unit were analysed.

Attribution of the sites sampled near the village of Moravské Lieskové (ML1, ML2) was problematic. The Albian age of the flysch rocks at the Bošáca and Moravské Lieskové villages has been demonstrated by their ammonite assemblages (Gross, 1963), but their attribution to any tectonic unit has been interpreted differently in the literature. Mišík et al. (1981) attributed these rocks to Fatricum, whereas Hók et al. (2009) considered this zone as part of the Klape Unit. The low amount of pebbles and their small average size (Mišík et al., 1981) point rather to a Fatric provenance for this zone.

Most of the samples were point samples from scree, because the flysch units examined very rarely form stable exposures, and only about four exposures were found for sampling (Fig. 3).

The petrographic composition of every sample (planimetric analysis) was determined by ribbon-counting in a polarizing microscope. After thin section preparation, the samples $(2 \mathrm{~kg}$ on average) were crushed, washed and sieved to obtain the $0.08-1 \mathrm{~mm}$ fraction. The heavy fraction was then separated by heavy liquids (bromoform, sodium polytungstate, with densities of $\sim 2.8$ ). The 0.08 to $0.25 \mathrm{~mm}$ fraction was studied in transmitted light, and percentage ratios of translucent heavy mineral assemblages were determined by ribbon point counting. The number of mounts for counting varied, and therefore a minimum of 300 grains were counted. Less translucent chrome-spinels were counted under reflected light using the same mounts as for the other minerals. This provided their real number in the samples examined, as well as excluding the omission of a part of them due to their semiopaque character. We are aware that this may cause problems when comparing percentage data with the findings from researchers who used solely transmitted light. Nevertheless, such data are generally not comparable, since the number of thinner Cr-spinel grains seen in transmitted light reveals nothing about their real amount.

Selected minerals, such as tourmalines, spinels, garnets, pyroxenes and blue amphiboles were hand-picked, embedded in epoxy resin, polished and coated with carbon. Their chemical compositions were analysed using a JEOL JXA-8530FE microprobe (Earth Science Institute of the Slovak Academy of Sciences in Banská Bystrica, Slovakia) under the following conditions: accelerating voltage $15 \mathrm{kV}$, sample current $20 \mathrm{nA}$, probe diameter 2-5 $\mu \mathrm{m}$, counting time $10 \mathrm{~s}$ - peak and $5 \mathrm{~s}$ for background, ZAF correction. The standards used, including lines and detection limits (in ppm) were: $\mathrm{Ca}(\mathrm{K} \alpha, 19-21)$ - diopside,

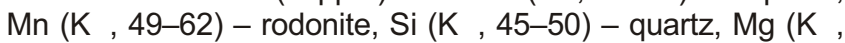
35-37) - olivine, F (Ko, 112-294) - fluorite, $\mathrm{Na}(\mathrm{K} \alpha, 31-36)$ -

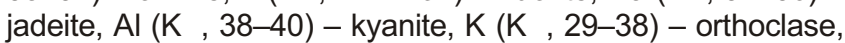
$\mathrm{Fe}(\mathrm{K} \alpha, 43-57)$ - hematite, $\mathrm{Ti}(\mathrm{K} \alpha, 35-38)$ - rutile, $\mathrm{Cr}-(\mathrm{K} \alpha$, 71-130) $-\mathrm{Cr}_{2} \mathrm{O}_{3}, \mathrm{Cl}(\mathrm{K} \alpha, 27-34)$ - tugtupite. A small amount of samples was also analysed using a CAMECA SX-100 electron microprobe at the State Geological Institute of Dionýz Štúr in Bratislava under similar conditions.

\section{RESULTS AND INTERPRETATIONS}

\section{PETROGRAPHIC ANALYSIS}

The sandstone samples analysed may be classified as fineto medium-grained psammites. Their grains are angular, subangular, with low to high sphericity (Pettijohn, 1987), mostly displaying very good sorting, without any preferred orientation. The main components are grains of quartz, lithic grains and matrix. Feldspars are rare, represented mostly by K-feldspars (e.g., microcline) and albite. Among the lithic grains, the most common are mica-schists (Fig. 4A), quartzites (Fig. 4B), basaltic volcanic rocks (Fig. 4C) and carbonates (Fig. 4D). Slightly recrystallized cherts and phyllites are less common. Bioclasts, e.g. foraminiferal tests and coralline algae are rare. The matrix of the sandstones is calcareous, representing from 0 to locally up to $50 \mathrm{vol} . \%$ of the rock. Some samples represented sandy limestones, and these were not included in the planimetric eval- 


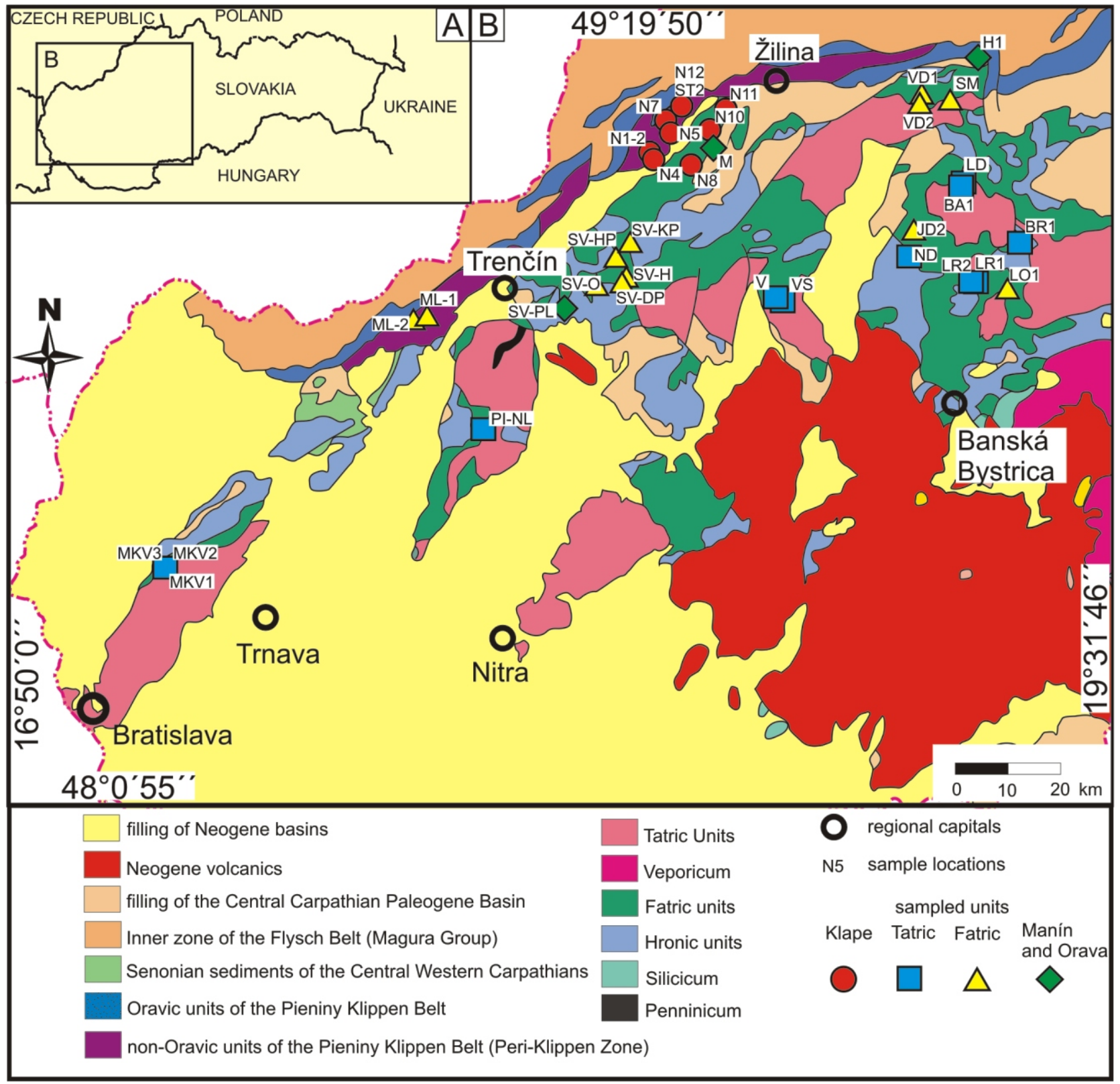

Fig. 2. Geological schematic map of western Slovakia and the location of the sites sampled

uation. The amount of matrix is high, mostly represented by calcareous cement. Therefore, the sandstones examined are mainly calcareous sandstones rather than wackes.

The prevailing fine-grained detrital fraction of the sandstones does not allow for a full interpretation of the primary rock composition from which the detrital material was derived. The only discernible lithic fragments are those of the fine-grained rocks. Most of the coarser-grained rocks were disaggregated into individual sand-sized grains.

According to the modal composition diagram published by Pettijohn et al. (1987), the sandstone samples analysed belong mostly to litharenites and sublitharenites, extending into the quartz arenite field (Fig. 5). The Klape Unit displays a relatively equal and narrow dispersion of the quartz/lithic fragments ratio, which plots exclusively in the litharenitic field. Samples from the Tatric and Fatric units (Poruba Formation) show wider dispersion of the ratios, since they are shifted towards the sub- litharenite and quartz arenite fields. Samples from the Manín Unit are located in a similar position to those of the Poruba Formation, whereas the sample from the Orava Unit is in the middle, just like the samples from the Klape Unit. In the provenance diagram published by Dickinson (1985), all the sandstones analysed fall into the recycled orogen field (Fig. 6).

\section{ABUNDANCE OF HEAVY MINERALS}

Counting of the heavy minerals (Fig. 7 and Table 1) showed that in most units, the predominant minerals are chrome-spinels, zircon, tourmaline and apatite. However, their percentages vary. Garnet is generally less commonly represented (usually $<11 \%$ ), but in the samples from the L'ubochnianska Valley (Tatricum, sample LD) and Medziholie Saddleback (Fatricum, sample SM), its percentage ranges up to 78 and $73 \%$. The apatite content in the sample from Záskalie (Manín 

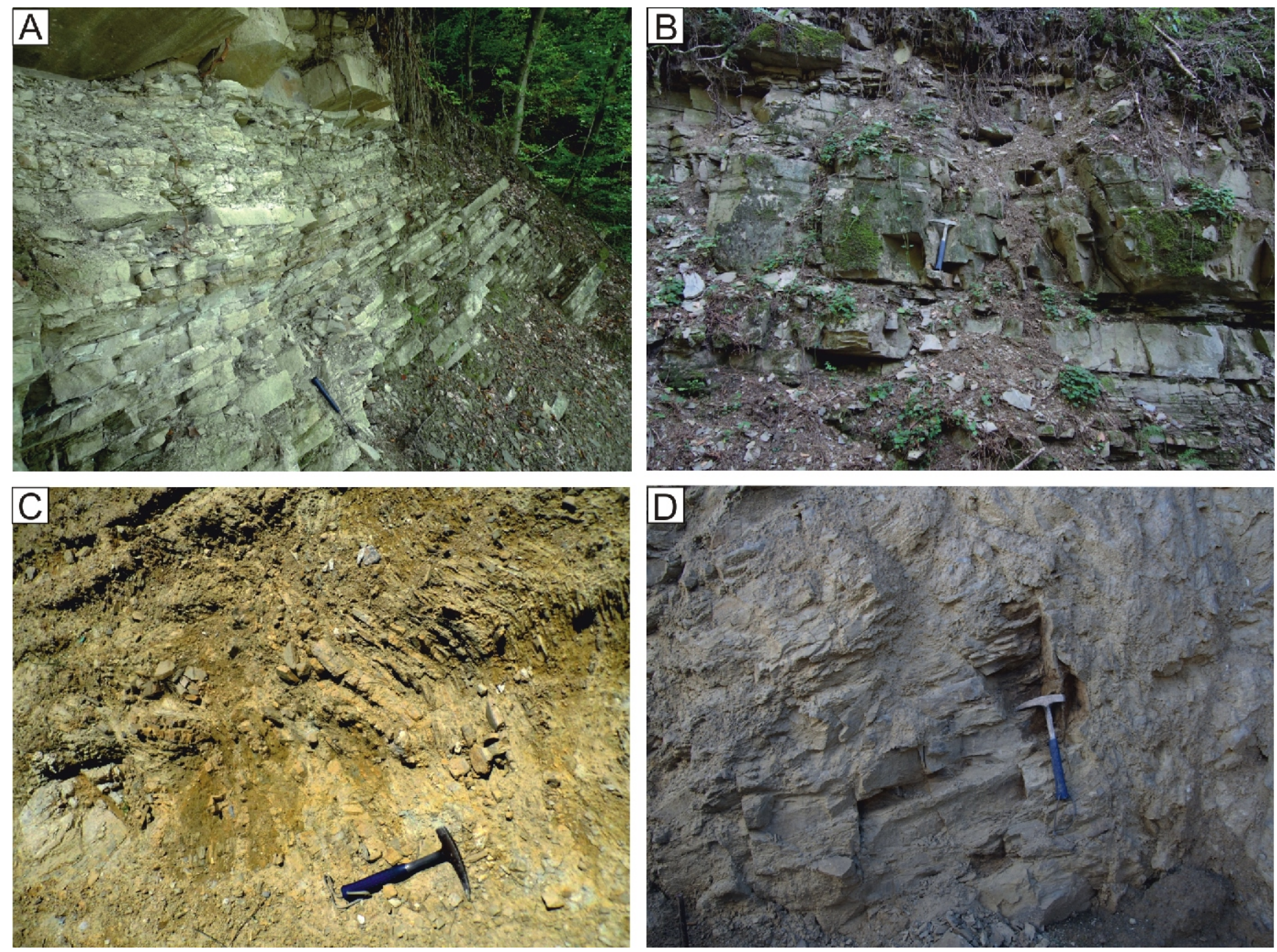

Fig. 3. View of some selected exposures of the formations sampled

A - exposure of thin-bedded turbidites of the Uhry Formation, locality N1 - above the railway at the Nosice Dam, Klape Unit; B - turbiditic sandstones of the Tatric Poruba Formation of varying thickness, locality BR1 - valley towards the Brankovo Waterfall, Low Tatra Mts.; C thin-bedded sandstone turbidites of the Tatric Poruba Formation in a field road near Liptovské Revúce, locality LR2; D - thin-bedded sandstone turbidites of the Cenomanian Praznov Formation, Záskalie locality near Manín Gorge, Manín Unit

Unit, sample M) is $58.6 \%$. In some samples, sphene, kyanite, monazite and epidote occur in relatively small amounts. At Havranský vrch Hill (Orava Unit, sample H), the amount of kyanite reaches $13.1 \%$. Sillimanite and staurolite appear less frequently. In several instances, blue amphiboles and pyroxenes occur in significant amounts. At the Predmier locality (Klape Unit, sample N11), blue amphiboles form $>20 \%$; at the Balcová (Tatricum, sample BA1) and Liptovská Osada (Fatricum, sample LO) localities, there were increased amounts of pyroxenes (>45\%), as well as at the Malé Karpaty Mts. Vývrat locality (Tatricum, sample MKV $->10 \%$ ). Chloritoid is present in some samples (e.g., Uhry, sample M5), and it was also found in the exotic flysch of the Eastern Alps (Woletz, 1963; Von Eynatten and Gaupp, 1999; Wagreich, 2003). Baryte, which occurred in some samples, e.g. from Uhry (N5), Balcová (BA1), Stupné (ST2), Havranský vrch $(\mathrm{H})$ and Jasenská Valley (JD2), was also not included in the heavy mineral percentages, because it is most likely of authigenic origin and its high percentages would distort the heavy-mineral spectra used for provenance analysis. No significant differences between the percentual ratios of heavy minerals were observed among the tectonic units, except for a slightly increased content of chrome-spinel in the Klape Unit versus a slightly higher ratio of zircon in the Poruba Formation.
The ZTR index of the samples examined (proportion of the ultrastable zircon-tourmaline-rutile trinity; Hubert, 1962) is relatively moderate, but along with the chrome-spinels (Table 1), ultrastable minerals prevail ( $\mathrm{Cr}$-spinels are ultrastable, too; Morton and Hallsworth, 2007). The ratio is lower only in the samples with an exceptionally higher content of garnet, pyroxene or apatite. However, the high amount of ultrastable minerals cannot be simply interpreted in terms of reworking from older strata. The high input of Cr-spinels is a new element in the Western Carpathians; in the previous detrital event in the Early and Middle Jurassic, only sialic material prevailed in the Pieniny Klippen Belt and the Central West Carpathian units (Aubrecht, 2001). It is then reasonable to assume that these $\mathrm{Cr}$-spinels were predominantly derived from a new, primary ophiolitic source that emerged during the Cretaceous.

Statistical linear regression analysis of the individual pairs of heavy minerals (Table 2) shows good mutual correlation only between garnet and staurolite. These were most likely derived from metamorphic sources. However, regression analysis of so few grains has low validity. The ZTR minerals (zircon-tourmaline-rutile) show a correlation coefficient (Pearson correlation coefficient $-R$ ) varying from 0.56 to 0.62 . The correlation indicates that at least part of the ZTR minerals, which are ultrastable ones and so most resistant against weathering, 

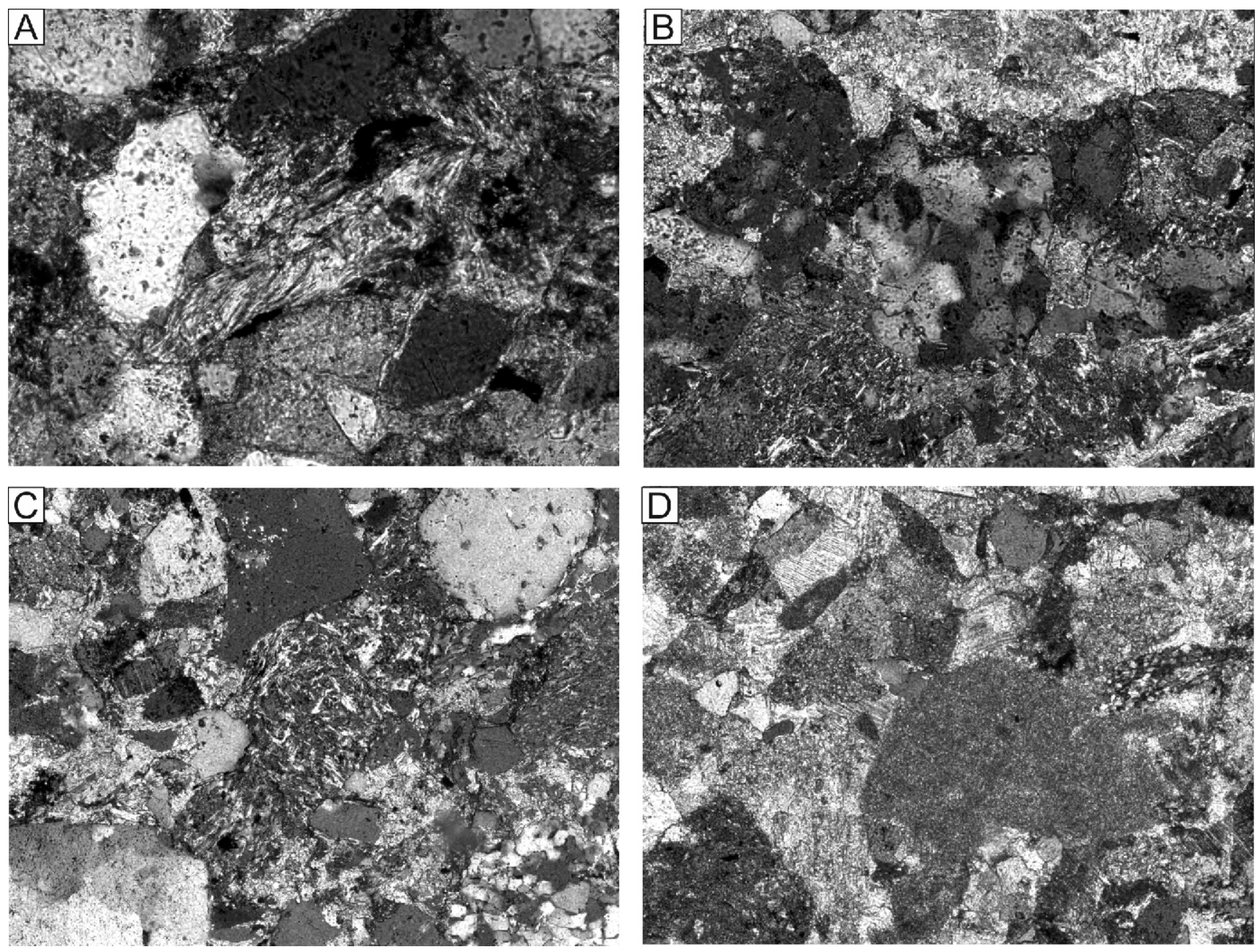

Fig. 4. Some examples of lithic fragments in the samples analysed

A - clast of mica-schist; B - lithoclast of quartzite; both sample N8, Považská Bystrica, Klape Unit; C - clast of basalt, sample N10, Plevník, Klape Unit; D - carbonate clast, sample from Stupné, Klape Unit

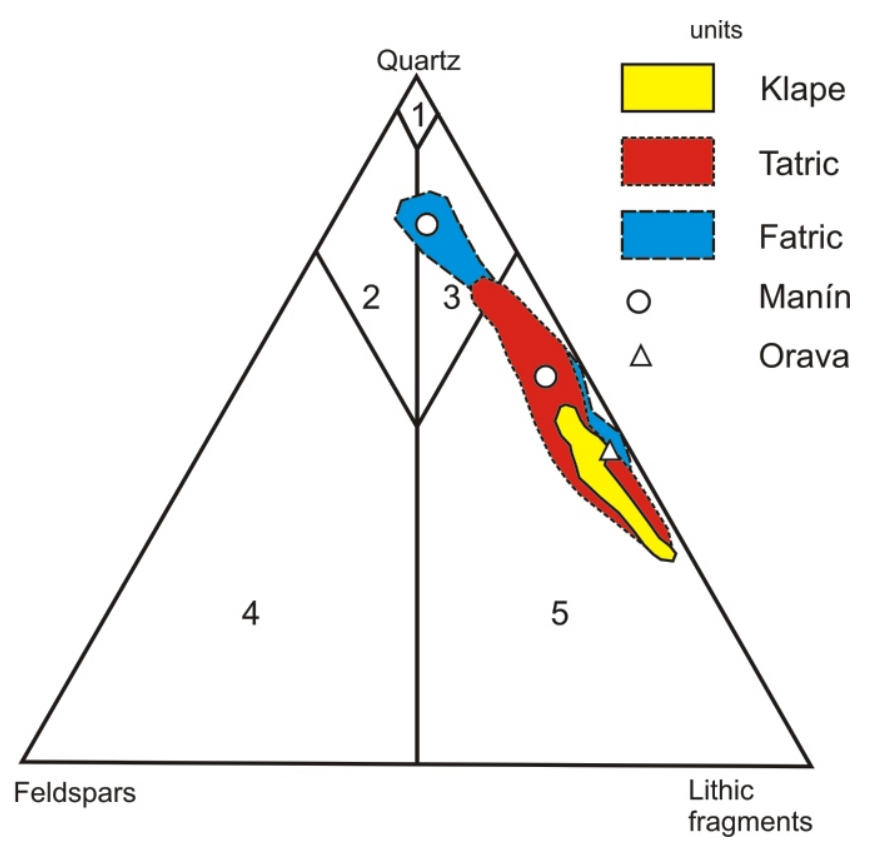

transport and intrastratal dissolution (see Hubert, 1962), were most likely derived from a common source represented by older deposits or low-grade metamorphosed sediments. The correlation of spinels with the ZTR trinity is generally weak; only tourmaline shows a moderate correlation (0.58). This means that at least part of the tourmaline may also be derived from ophiolites. Other heavy-mineral couples show no mutual correlation or even a slightly negative one. Heavy mineral spectra thus point to a complex and variable source area, dominated by older sediments and ophiolites. Primary metamorphic and magmatic sialic rocks were unevenly distributed and relatively rare. In some cases, an eventual better primary correlation (e.g., between blue amphiboles and Cr-spinels) was later erased by a strongly different resistance of the individual minerals during transport and diagenesis processes.

Fig. 5. Plot of the petrographic composition obtained by point-counting in the sandstone classification diagram of Pettijohn et al. (1987)

Although the sandstones examined often contain $>15 \%$ of matrix, it is calcareous and they do not belong to wackes; 1 - quartz arenite, 2 - subarkose, 3 - sublitharenite, 4 - arkosic arenite, 5 - litharenite 


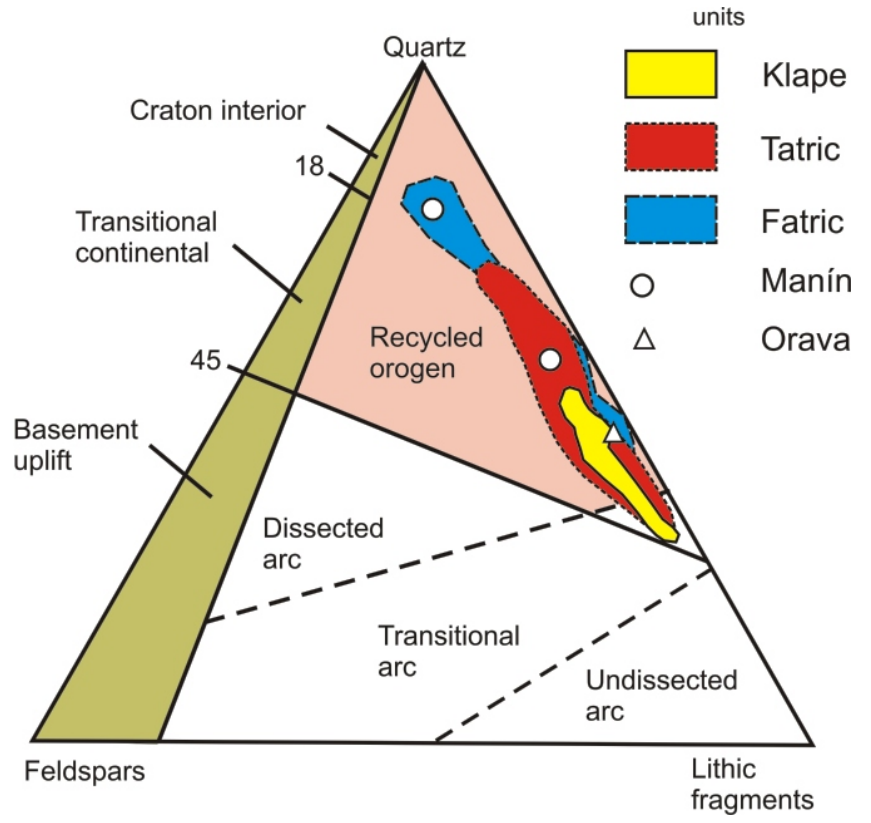

Fig. 6. Plot of the petrographic composition obtained by point-counting in the arenite provenance diagram by Dickinson (1985)
CHEMICAL COMPOSITION OF DETRITAL SPINEL AND ITS ORIGIN

The spinel fragments analysed were homogeneous and unzoned. The spinel grains commonly contain small vugs caused by dissolution of unstable inclusions (e.g., pyroxene and olivine) during weathering (Bellová et al., 2018; Fig. 3A). No measurable inclusions were preserved. One grain from the Uhry locality had an alteration rim (Bellová et al., 2018; Fig. 3B).

In diagnosing different types of spinel, chemical variability in elements such as $\mathrm{Mg}, \mathrm{Fe}, \mathrm{Cr}, \mathrm{Al}$ and $\mathrm{Ti}$ is important. From the calculated IMA-approved end-member species, the most common were $\mathrm{Cr}$-rich spinel, chromite and magnesiochromite members (for representative analyses see Bellová et al., 2018). For the purpose of provenance, two types of diagram are used. The first diagram, $\mathrm{Mg} /\left(\mathrm{Mg}+\mathrm{Fe}^{2+}\right)$ vs. $\mathrm{Cr} /(\mathrm{Cr}+\mathrm{Al})$ was introduced by Dick and Bullen (1984). It distinguishes three fields indicating various types of ophiolite: (1) Type I ophiolites representing peridotites for which $\mathrm{Cr} /(\mathrm{Cr}+\mathrm{Al})$ is $<0.60$ (mid-oceanic ridge peridotites), (2) Type III ophiolites representing peridotites with spinels having $>0.60 \mathrm{Cr} /(\mathrm{Cr}+\mathrm{Al})$, representing the early stages of arc formation, (3) Type II ophiolites bearing spinels with a wide range of $\mathrm{Cr} /(\mathrm{Cr}+\mathrm{Al})$, representing transitional phases. Herein, we use the modified diagram of Pober and Faupl (1988) to distinguish spinels derived from harzburgites, Iherzolites, podiform chromitites and cumulates (Fig. 8). Most of the grains match the harzburgitic field, with some overlap over the fields of podiform chromitites and cumulates. Some spinels

Spl $\square$ Zrn $\square$ Tur $\square$ Ap $\square$ Rt $\square$ Grt $\square$ Mnz $\square$ Spn $\square$ St $\square$ Ky $\square$ Px $\square$ Amp $\square$ B.Amp $\square$ Ep $\square$ Sil

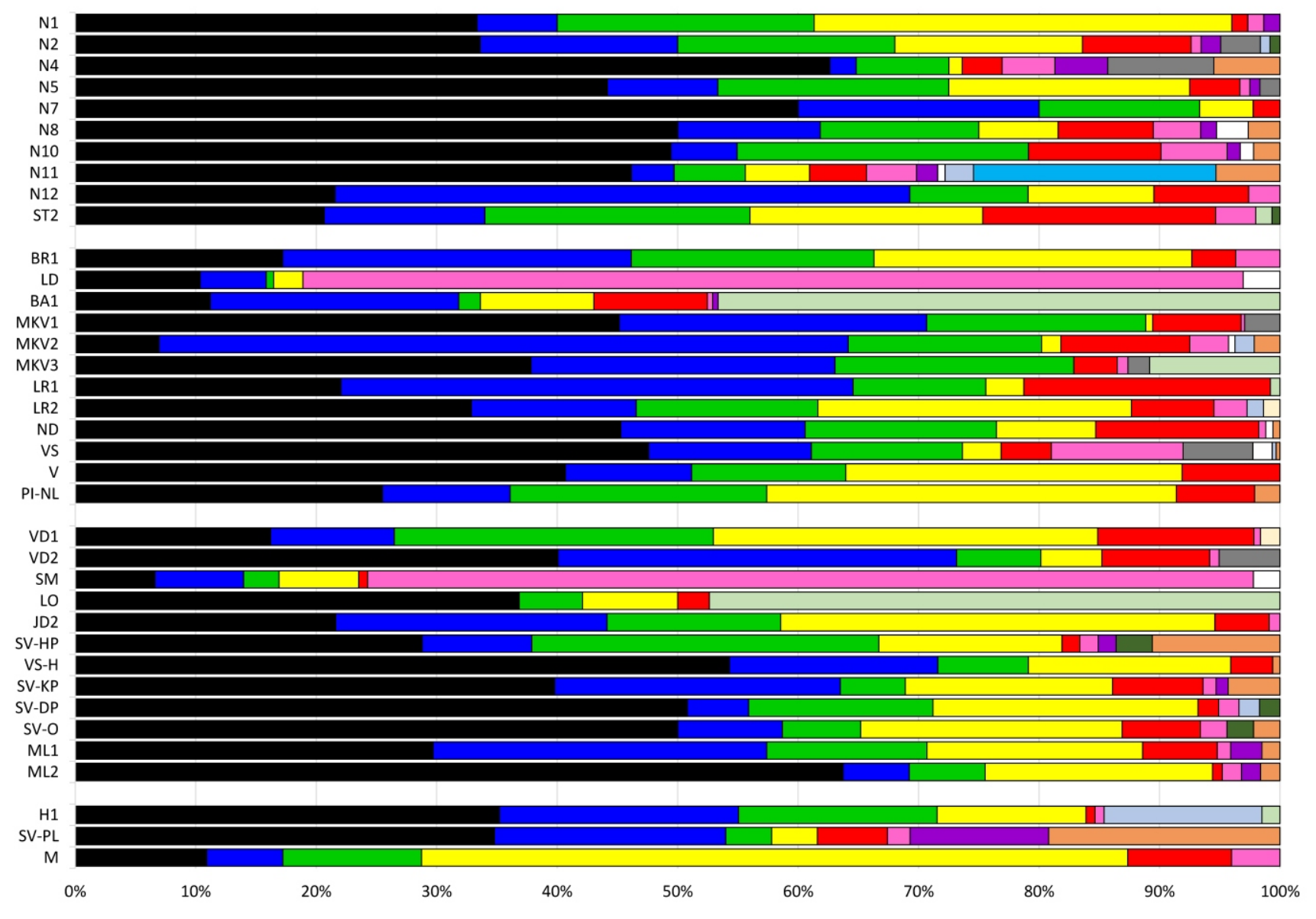

Fig. 7. Percentage ratios of the heavy minerals in the samples analysed

Mineral abbreviations (from Whitney and Evans, 2010): Amp - amphibole, Ap - apatite, B-Amp - blue amphibole, Ep - epidote, Grt - garnet, Ky - kyanite, Mnz - monazite, Px - pyroxene, Rt - rutile, Sil - sillimanite, Spl - spinel, Spn - titanite, St - staurolite, 


\begin{tabular}{|c|c|c|c|c|c|c|c|c|c|c|c|c|c|c|c|c|c|c|c|c|c|c|c|c|}
\hline & 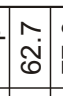 & $\begin{array}{lll}0 \\
\hat{N} \\
\end{array}$ & $\begin{array}{c}\infty \\
\infty \\
0\end{array}$ & & & ; & & & & & 0 & & & & & & & & & $\bar{v}$ & & & - & \\
\hline $\bar{N}$ & 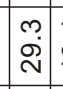 & 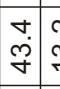 & 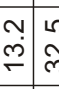 & & & & 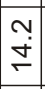 & & & & \ulcorner & & & & & & & & & & & & $\begin{array}{l} \\
\dot{g}\end{array}$ & \\
\hline$\overline{\bar{n}}$ & 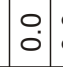 & - & 0. & & & & 0 & & & & & & & & & & & & & & & & 号 & \\
\hline Ш & 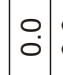 & 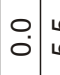 & is & & & & & & & & & & & & & & & & & & & & b & \\
\hline & 8 & \begin{tabular}{ll}
0 \\
\hdashline
\end{tabular} & : & & & $0^{\circ}$ & $\check{\text { Dे }}$ & & & & & & & & & & & & & & & & : & \\
\hline$\frac{\mathrm{E}}{\mathrm{z}}$ & c & $\begin{array}{l}\infty \\
0 \\
0\end{array}$ & 0 & & & 0 & ㅇ. & & tó & & $\stackrel{\circ}{\circ}$ & & & & & & & & & & & & $p$ & \\
\hline a & 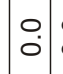 & $\begin{array}{l}0 \\
0 \\
0\end{array}$ & 0. & & & $\begin{array}{l}0 \\
0\end{array}$ & $\begin{array}{l}0 \\
0 \\
0\end{array}$ & 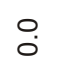 & & & & & & & & & & & & & & & : & \\
\hline$\vec{x}$ & & $\infty$ & 0.0 & & & & $\stackrel{\vec{N}}{\stackrel{N}{*}}$ & & & & & & & & & & & & & & & & : & \\
\hline 范 & c & 0 & 0. & c & & -1 & \begin{tabular}{|l|} 
\\
\end{tabular} & & & & & & & & & & & & & & & & : & \\
\hline ஸे & $0^{\circ}$ & $\stackrel{m}{m}$ & $\infty$. & & & & $\begin{array}{l}0 \\
\end{array}$ & & & & & & & & & & & & & & & & & \\
\hline$\sum_{\Sigma}^{N}$ & $\stackrel{m}{-}$ & & $\stackrel{+}{+}$ & & & 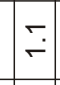 & $\stackrel{\infty}{\leftarrow}$ & & & & & & & & & & & & & & & & •? & \\
\hline 0 & 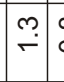 & 0 & $\dot{\nabla}$ & & & ?? & $\bar{f}_{\dot{f}}$ & & & & & & & & & & & & & & & & & \\
\hline צ & $\stackrel{\text { s. }}{-}$ & $\begin{array}{l}\circ \\
\dot{a}\end{array}$ & m. & & & $=$ & F & & & & $\circ$ & & & & & & & & & & $?$ & & 's & \\
\hline$\varangle$ & লे & 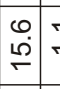 & 4 & & & 10 & is & & & & & & & & & 户্ & & & & & & & $\dot{\sigma}$ & \\
\hline & 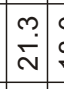 & \ulcorner & $\begin{array}{ll}\hat{\sim} & \sigma \\
g\end{array}$ & -5 & & $\mid \begin{array}{c}N \\
\dot{N} \\
\stackrel{N}{*}\end{array}$ & & & & & 0 & & & & & & & & & & & & o. & \\
\hline$N$ & $c$ & 4 & & ¿ & & 10 & & & & & & & & & & & & & & & & & & \\
\hline c) & 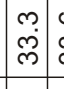 & 0 & $\begin{array}{l}0 \\
\stackrel{1}{ن}\end{array}$ & : & & 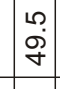 & $\tilde{c}$ & & & & & & & & & & & & & & & & $\dot{b}^{\circ}$ & \\
\hline 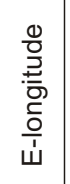 & ลี & 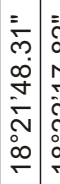 & $\tilde{\sigma}$ & 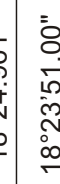 & 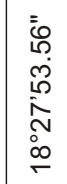 & $\left|\begin{array}{l}0 \\
0 \\
0 \\
0 \\
0 \\
0\end{array}\right|$ & & $\begin{array}{l}0 \\
0 \\
0\end{array}$ & 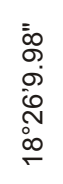 & 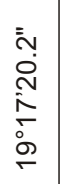 & $\stackrel{\tilde{\alpha}}{\alpha}$ & 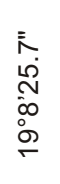 & $\begin{array}{l}\bar{z} \\
4 \\
0 \\
\vdots \\
\vdots \\
\vdots \\
\vdots\end{array}$ & $\stackrel{\text { }}{\stackrel{N}{N}}$ & & $\stackrel{+}{\stackrel{+}{\circ}}$ & & & & & 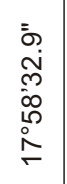 & t? & 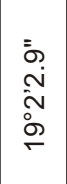 & . \\
\hline
\end{tabular}

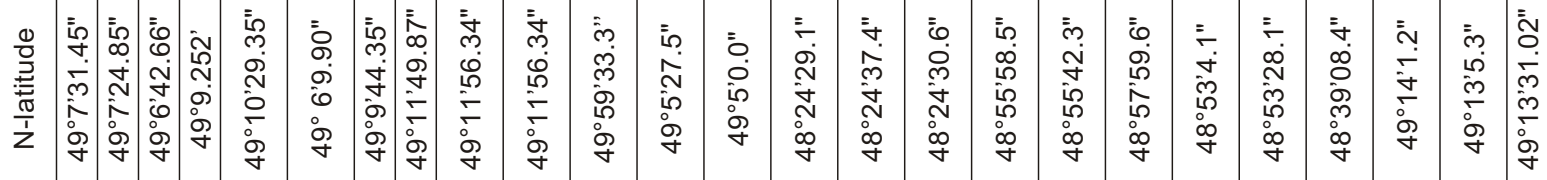

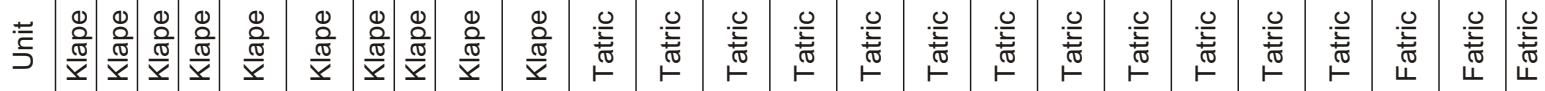

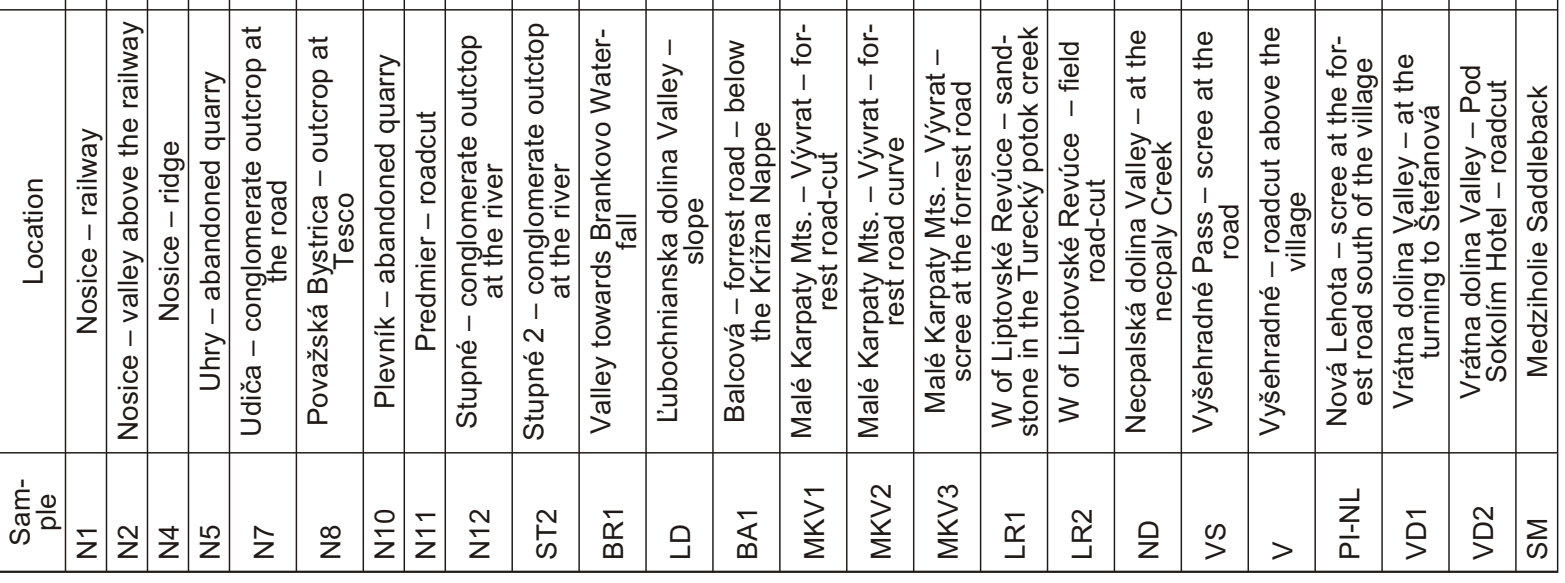




\begin{tabular}{|c|c|c|c|c|c|c|c|c|c|c|c|c|}
\hline 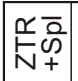 & \begin{tabular}{|c|}
\multirow{f}{*}{} \\
\end{tabular} & $\bar{\varnothing}$ & \begin{tabular}{|l|}
$N$ \\
$\infty$ \\
0 \\
0
\end{tabular} & \begin{tabular}{|l|}
\multirow{i}{*}{} \\
$\stackrel{\infty}{\infty}$
\end{tabular} & 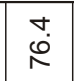 & 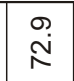 & $\frac{\hat{i}}{\grave{i}}$ & \begin{tabular}{|l|}
$\begin{array}{l}0 \\
0 \\
i\end{array}$ \\
\end{tabular} & 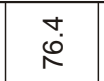 & $\begin{array}{l}m \\
\stackrel{N}{\mathrm{~N}}\end{array}$ & $\begin{array}{l}0 \\
\ddot{0} \\
0\end{array}$ & ì \\
\hline$\frac{\underline{r}}{N}$ & $\stackrel{?}{\sim}$ & $\begin{array}{l}\vec{r} \\
\dot{q}\end{array}$ & ণ্ল & 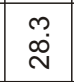 & $\begin{array}{l}\dot{0} \\
\dot{\phi} \\
\dot{m}\end{array}$ & $\overline{\mathrm{N}}$ & $\hat{\grave{n}}$ & \begin{tabular}{|c|}
\multirow{4}{*}{} \\
\end{tabular} & 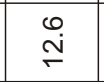 & \begin{tabular}{|c|}
$\overline{\hat{m}}$ \\
\end{tabular} & $\begin{array}{l}\infty \\
\stackrel{\infty}{N} \\
\stackrel{\infty}{n}\end{array}$ & \\
\hline$\overline{\bar{\omega}}$ & $\stackrel{\circ}{\circ}$ & $\stackrel{\circ}{\circ}$ & $\stackrel{\circ}{\circ}$ & $\stackrel{\circ}{\circ}$ & $\stackrel{\circ}{\circ}$ & $\stackrel{\circ}{\circ}$ & $\begin{array}{l}0 \\
0\end{array}$ & $\stackrel{\circ}{\circ}$ & $\stackrel{\circ}{\circ}$ & $\begin{array}{l}0 \\
0\end{array}$ & $\stackrel{\circ}{\circ}$ & 10 \\
\hline யி & $\stackrel{\circ}{\circ}$ & ㅇ. & $\begin{array}{l}0 \\
\stackrel{0}{\circ}\end{array}$ & $\begin{array}{l}\bullet \\
\end{array}$ & $\begin{array}{l}m \\
\dot{\sigma}\end{array}$ & $\stackrel{\circ}{\circ}$ & $\stackrel{N}{N}$ & $\stackrel{\sim}{\circ}$ & $\stackrel{\leftrightarrow}{r}$ & $\begin{array}{l} \\
\end{array}$ & $\begin{array}{l}\text { Na } \\
\text { O }\end{array}$ & c \\
\hline 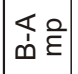 & O. & $\stackrel{\circ}{\circ}$ & $\stackrel{\circ}{\circ}$ & $\stackrel{\circ}{\circ}$ & $\stackrel{\circ}{\circ}$ & $\stackrel{\circ}{\circ}$ & $\because$ & $\stackrel{\circ}{\circ}$ & $\stackrel{\circ}{\circ}$ & O. & $\stackrel{\circ}{\circ}$ & O \\
\hline$\frac{\mathrm{g}}{\mathrm{\xi}}$ & $\stackrel{\circ}{\circ}$ & $\stackrel{\circ}{\circ}$ & $\stackrel{\circ}{\dot{m}}$ & $\stackrel{0}{\circ}$ & $\because$ & 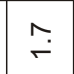 & $\underset{\sim}{N}$ & $\stackrel{0}{\circ}$ & $\because$ & $\begin{array}{l}0 \\
0\end{array}$ & $\stackrel{0}{\circ}$ & O. \\
\hline x & 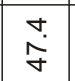 & 0. & $\circ$ & $\stackrel{\circ}{\circ}$ & $\stackrel{\circ}{\circ}$ & $\stackrel{\circ}{\circ}$ & $\stackrel{0}{0}$ & $\stackrel{\circ}{\circ}$ & $\stackrel{\circ}{\circ}$ & \begin{tabular}{|l|l|} 
\\
\end{tabular} & $\stackrel{\circ}{\circ}$ & Oa \\
\hline$\vec{x}$ & $\stackrel{\circ}{\circ}$ & $\stackrel{\circ}{\circ}$ & 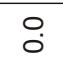 & $\begin{array}{l}\circ \\
\circ\end{array}$ & $\begin{array}{l} \\
\circ\end{array}$ & $\stackrel{?}{\circ}$ & $\begin{array}{l}0 \\
\end{array}$ & $\because$ & $\stackrel{\circ}{\circ}$ & \begin{tabular}{|l|}
$\overline{\dot{m}}$ \\
\end{tabular} & $\stackrel{\circ}{\circ}$ & 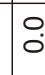 \\
\hline ஸ & $\stackrel{\circ}{\circ}$ & $\stackrel{\circ}{\circ}$ & $\stackrel{\circ}{\circ}$ & $\stackrel{\circ}{\circ}$ & $\stackrel{\circ}{\circ}$ & $\stackrel{\circ}{\circ}$ & 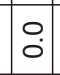 & $\stackrel{\circ}{\circ}$ & $\stackrel{\circ}{\circ}$ & $\begin{array}{l}0 \\
0\end{array}$ & $\stackrel{\circ}{\circ}$ & $\stackrel{\circ}{\circ}$ \\
\hline के & $\stackrel{\circ}{\circ}$ & $\stackrel{\circ}{\circ}$ & $\stackrel{\circ}{\circ}$ & $\stackrel{\circ}{\circ}$ & $\stackrel{\circ}{\circ}$ & $\stackrel{\circ}{\circ}$ & $\because$ & $\stackrel{\circ}{\circ}$ & $\stackrel{\circ}{\circ}$ & $\begin{array}{l} \\
\end{array}$ & $\stackrel{\circ}{\circ}$ & 웅 \\
\hline$\sum_{\sum}^{N}$ & O. & O. & 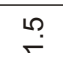 & $\circ$ & $\stackrel{\circ}{r}$ & $\stackrel{\circ}{\circ}$ & $\stackrel{\circ}{\circ}$ & $\stackrel{\bullet}{\sim}$ & $\stackrel{\circ}{\circ}$ & ○. & 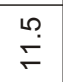 & ○. \\
\hline 志 & $\because$ & $\stackrel{\Omega}{0}$ & $\stackrel{\text { L }}{\longrightarrow}$ & $\stackrel{\circ}{\circ}$ & $\check{r}$ & $\stackrel{\overbrace{}}{-}$ & $\stackrel{\sim}{N}$ & $\check{r}$ & $\stackrel{\circ}{-}$ & $\hat{o}$ & $\stackrel{\leftrightarrow}{r}$ & $\stackrel{\circ}{\dot{r}}$ \\
\hline $\overrightarrow{\mathbb{x}}$ & $\stackrel{\leftrightarrow}{\mathrm{N}}$ & 10 & $\stackrel{\text { Pִ }}{\leftarrow}$ & 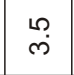 & $\stackrel{\stackrel{\rho}{r}}{\wedge}$ & $\stackrel{\overbrace{}}{-}$ & $\begin{array}{l}0 \\
0 \\
0\end{array}$ & ํ. & $\stackrel{\infty}{\circ}$ & $\hat{\circ}$ & $\stackrel{\infty}{\infty}$ & $\begin{array}{l}0 \\
\infty \\
\end{array}$ \\
\hline$\frac{2}{4}$ & $\stackrel{\rho}{r}$ & $\begin{array}{l}\circ \\
\dot{\rho}\end{array}$ & $\begin{array}{l}\text { Nָ } \\
\stackrel{2}{\circ}\end{array}$ & $\begin{array}{l}\infty \\
\dot{0} \\
\stackrel{0}{0}\end{array}$ & 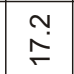 & 오 & $\stackrel{\grave{n}}{\dot{N}}$ & 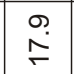 & $\begin{array}{l}\stackrel{\sigma}{\infty} \\
\stackrel{\infty}{\infty}\end{array}$ & $\begin{array}{l}\stackrel{\sim}{\mathrm{i}} \\
\stackrel{2}{ }\end{array}$ & $\stackrel{\infty}{\infty}$ & $\begin{array}{l}0 \\
0 \\
\infty \\
i s\end{array}$ \\
\hline$\risingdotseq$ & $\overbrace{i}^{m}$ & $\begin{array}{l}\stackrel{\stackrel{J}{*}}{r} \\
\end{array}$ & $\begin{array}{l}\infty \\
\stackrel{\infty}{\infty} \\
\stackrel{\infty}{n}\end{array}$ & 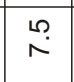 & 苑 & $\begin{array}{l}m \\
\stackrel{m}{\sim} \\
\stackrel{\rho}{n}\end{array}$ & $\begin{array}{l}0 \\
0 \\
0\end{array}$ & 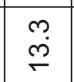 & $\overbrace{0}$ & \begin{tabular}{|l|l|}
\multicolumn{1}{|c}{} \\
0 \\
0
\end{tabular} & $\stackrel{\infty}{\infty}$ & $\frac{5}{7}$ \\
\hline$\stackrel{\Xi}{N}$ & $\stackrel{\circ}{\circ}$ & $\stackrel{\sim}{\mathrm{N}}$ & $\dot{\sigma}$ & $\stackrel{m}{\stackrel{m}{\sim}}$ & $\hat{\tilde{N}}$ & $\overline{i s}$ & $\hat{\infty}$ & $\hat{\stackrel{N}{N}}$ & مْ & $\mid \begin{array}{l}\mid \\
\dot{\sigma} \\
\dot{\sigma}\end{array}$ & 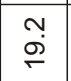 & @ं \\
\hline के & $\begin{array}{l}\infty \\
0 \\
\dot{m}\end{array}$ & 0 & $\begin{array}{l}\infty \\
\stackrel{\infty}{\infty} \\
\stackrel{\infty}{\infty}\end{array}$ & $\begin{array}{l}\text { m } \\
\text { İ }\end{array}$ & $\begin{array}{l} \\
\stackrel{\infty}{\infty} \\
\dot{m}\end{array}$ & $\begin{array}{l}\infty \\
0 \\
0 \\
i\end{array}$ & $\begin{array}{l} \\
\dot{0} \\
i\end{array}$ & $\hat{\stackrel{N}{N}}$ & 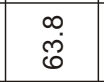 & \begin{tabular}{|c|}
\multirow{2}{*}{} \\
$\stackrel{\rho}{\rho}$
\end{tabular} & \begin{tabular}{|c|}
$\infty$ \\
$\dot{m}$ \\
\end{tabular} & $\begin{array}{l} \\
\\
\end{array}$ \\
\hline 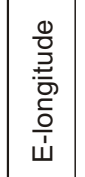 & 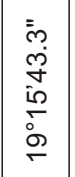 & 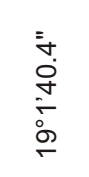 & 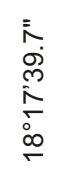 & 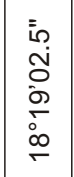 & 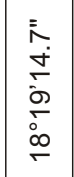 & 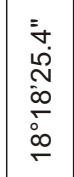 & 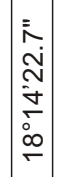 & 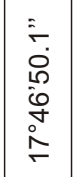 & 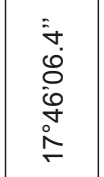 & 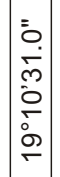 & $\mid \begin{array}{l}0 \\
0 \\
0 \\
0 \\
0 \\
0 \\
0 \\
- \\
0\end{array}$ & $\begin{array}{l}\overline{0} \\
0 \\
0 \\
0 \\
0 \\
\infty \\
\infty \\
\varnothing\end{array}$ \\
\hline 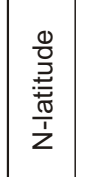 & $\mid \begin{array}{c}0 \\
0 \\
0 \\
i \\
i \\
0 \\
0 \\
o \\
+\infty\end{array}$ & 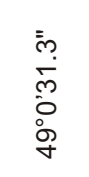 & 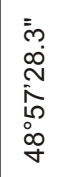 & 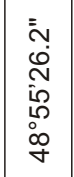 & $\begin{array}{c}\bar{z} \\
\dot{o} \\
o \\
o \\
o \\
0 \\
o \\
o \\
o\end{array}$ & 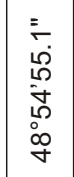 & 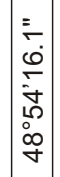 & $\begin{array}{l}0 \\
0 \\
\dot{0} \\
0 \\
0 \\
0 \\
o \\
o \\
o\end{array}$ & $\begin{array}{l}\bar{y} \\
\dot{0} \\
\dot{0} \\
\dot{0} \\
0 \\
0 \\
\infty \\
\dot{\sigma}\end{array}$ & 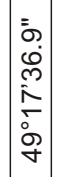 & 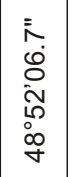 & $\begin{array}{l}m \\
\dot{0} \\
\dot{0} \\
\circ \\
\stackrel{8}{+}\end{array}$ \\
\hline$\overline{\mathrm{z}}$ & 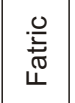 & 总 & 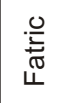 & 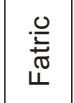 & $\begin{array}{l}\stackrel{0}{\underline{E}} \\
\stackrel{\omega}{\omega}\end{array}$ & 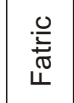 & 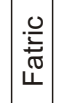 & 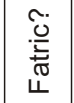 & 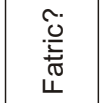 & 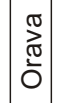 & 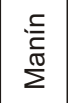 & $\mid \begin{array}{l}\frac{.5}{\bar{E}} \\
\sum\end{array}$ \\
\hline 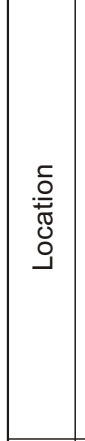 & 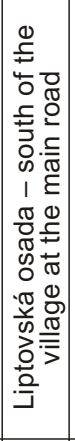 & 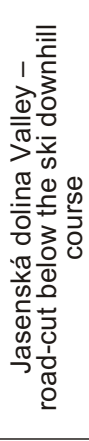 & 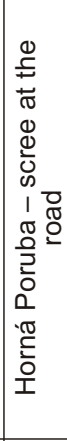 & 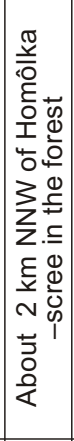 & 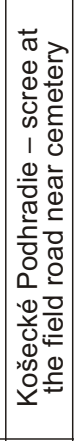 & 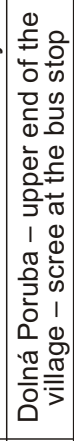 & 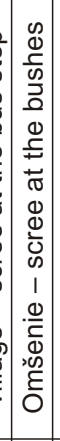 & 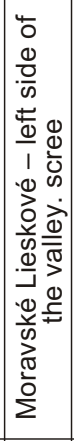 & 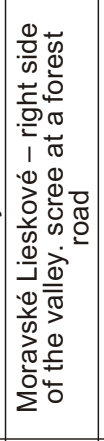 & 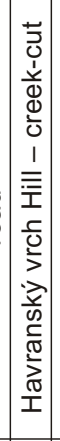 & 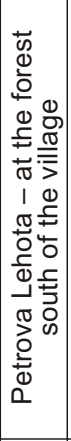 & 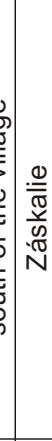 \\
\hline$\underline{\xi}$ & ○ & ֻี & क & 齐 & 辛 & 号 & 号 & $\bar{\Sigma}$ & $\stackrel{\sim}{\Sigma}$ & $\overline{\mathbf{I}}$ & 京 & \\
\hline
\end{tabular}


Ta ble 2

Results of statistical linear regression analysis of the individual pairs of heavy minerals

\begin{tabular}{|l|c|c|}
\hline $\begin{array}{c}\text { Mineral } \\
\text { pair }\end{array}$ & $\begin{array}{c}\text { Correlation } \\
\text { coefficient }(r)\end{array}$ & Degree of correlation \\
\hline Zrn-Ru & 0.62 & moderate positive correlation \\
\hline Zrn-Tur & 0.61 & moderate positive correlation \\
\hline Tur-Spl & 0.58 & moderate positive correlation \\
\hline Rt-Tur & 0.56 & moderate positive correlation \\
\hline Zrn-Spl & 0.45 & weak positive correlation \\
\hline Tur-Ap & 0.41 & weak positive correlation \\
\hline Rt-Spl & 0.34 & weak positive correlation \\
\hline Rt-Ap & 0.24 & no linear relationship \\
\hline Zrn-Ap & 0.13 & no linear relationship \\
\hline
\end{tabular}

outside the fields distinguished were most likely altered or metamorphosed. Only three analyses plotted on the non-overlapping part of the lherzolite field, having $\mathrm{Cr} /(\mathrm{Cr}+\mathrm{Al})<0.3$.

The second type of diagram used for purposes of provenance is the $\mathrm{Al}_{2} \mathrm{O}_{3}$ vs. $\mathrm{TiO}_{2}$ diagram introduced by Lenaz et al (2000) and Kamenetsky et al. (2001). This diagram is based on the fact that most mantle rock spinels have $\mathrm{TiO}_{2}<0.2 \mathrm{wt} . \%$. and most volcanic spinels have $\mathrm{TiO}_{2}>0.2 \mathrm{wt} . \%$ (for an overview. see Lenaz et al., 2009). This diagram indicates that most of the spinels were derived from supra-subduction zone peridotites the others, which have aluminium-depleted and higher-titanium chemistry, were most likely derived from volcanic arcs. The grains that plotted in the pure Iherzolite field in the previous diagram plot on the non-overlapping part of the MORB peridotites field (Fig. 9). The altered spinels have a low aluminium content and some of them plot outside any pre-defined fields of fresh magmatic spinels.

\section{CHEMICAL COMPOSITION OF DETRITAL TOURMALINE AND ITS ORIGIN}

Detrital tourmaline grains in the samples analysed have a brown to green, but mostly khaki-green colour. They are mostly subhedral, euhedral grains being rare. Observations of BSE images show that the tourmaline grains are predominantly unzoned, but some possess distinct zonation displaying a complex intergrowing pattern of two phases of tourmaline or tourmaline with quartz, thus attaining a complex zoned mosaic appearance (Fig. 10).

According to the classification diagrams of Henry et al. (2011), most of the tourmalines belong to the alkali and X-vacant groups (Fig. 11) with a schorl-dravitic, less foititic and magnesio-foititic composition with a higher proportion of X-site vacancies (Fig. 12). The discrimination diagrams of Henry and Guidotti (1985) revealed that most of the tourmaline grains were likely derived from metasedimentary rocks, i.e. from metapelites and metapsammites, either coexisting or not with an $\mathrm{Al}$-saturating phase; some were also derived from $\mathrm{Fe}^{3+}$-rich quartz-tourmaline rocks, calc-silicate rocks and metapelites (Fig. 13). The metasedimentary source rocks were mostly poor in Ca. There is also a group of tourmaline crystals (e.g., almost the entire sample from Havranský vrch Hill) which show compositions belonging to the field of Li-poor granitoid rocks and their associated pegmatites and aplites.

In the zoned crystals, the overgrowing zones mostly plot on the metasedimentary rock field, and in one grain, the overgrowing zone displayed a magnesium-depleted composition, thus corresponding to Li-poor granitoid rocks (Bellová et al., 2018; Fig. 10). Complexly zoned tourmaline grains represent two types: (1) grains with fabric consisting of several tourmaline phases chaotically arranged as laths, (2) grains displaying a fine intergrowth of tourmaline and quartz. The first type of tourmaline shows a continuous trend of $\mathrm{Al}$ and its substitution by $\mathrm{Fe}^{3+}$ at the $Y$ and $Z$ sites, thus shifting from a schorl-dravitic composition to bosiite. The second type predominantly consists of schorldravitic tourmaline, but with scarce occurrence of Al-enriched oxy-dravitic, foititic and magnesio-foititic compositions (for further details, see Aubrecht et al., 2020).

\section{CHEMICAL COMPOSITION OF DETRITAL BLUE AMPHIBOLES} AND THEIR ORIGIN

Blue amphiboles were found only at the Predmier locality. They are characterized by a rich blue colour, with pleochroism up to violet shades (Fig. 15A, B). The grains are mostly broken and subhedral.

The microanalyses showed that the amphiboles are sodic, with $\mathrm{B}(\mathrm{Na}+\mathrm{Li}) / \Sigma \mathrm{B} \geq 0.75, \mathrm{BNa} / \Sigma \mathrm{B} \geq \mathrm{BLi} / \Sigma \mathrm{B}$ (Hawthorne et al. 2012). Their formulas show that $\mathrm{Al}^{\mathrm{Vl}} \geq \mathrm{Fe}^{3+}$ and the majority of the amphiboles analysed plot on the field of glaucophane and some on ferroglaucophane (Leake et al., 1997; Fig. 14). On the basis of pressure estimation according to the sodium and aluminium contents (Brown, 1977), the amphiboles measured originated at pressures between 6 and $7 \mathrm{~kb}$, but closer to $7 \mathrm{~kb}$ (Bellová et al., 2018).

\section{CHEMICAL COMPOSITION OF DETRITAL PYROXENES} AND THEIR ORIGIN

Pyroxenes were found in greater amounts (>40\%) at the Liptovská Osada and Balcová localities; several grains were found at the Vývrat locality $(>10 \%)$. Small amounts $(>1 \%)$ were also found at the Havranský vrch and Stupné localities. The pyroxene grains are mostly of greenish-brown colour, with faint pleochroism, broken, mostly subhedral, but fully euhedral grains are also common (Fig. 15C, D). According to $\mathrm{Ca}$, Fe and Mg ratios (Fig. 16), most are classified as enstatite; nine grains were in the fields of augite and one of diopside (Morimoto et al., 1988).

\section{CHEMICAL COMPOSITION OF DETRITAL GARNET AND ITS ORIGIN}

The garnet grains were transparent and colourless to pale pink under the microscope; they represented mainly fragments of crystals without preserved crystal faces or zonation. Garnets from six localities were analysed (Fig. 17). Most of the detrital garnet grains examined showed a clear dominance of almandine molecules (up to nearly $74 \%$ ); several grains possessed higher pyrope contents (>25\% -4 grains), thus attaining a pyrope-almandine composition.

\section{ZIRCON MORPHOLOGICAL TYPOLOGY}

The vast majority of zircon grains were rounded and anhedral, indicating that they were predominantly reworked from older clastic rocks. Only 35 euhedral zircon crystals could be selected for zircon typology analysis (Pupin and Turco, 1972; Pupin, 1980) from the localities of Stupné, Liptovské Revúce, Havranský vrch, Moravské Lieskové and Brankovo Waterfall. Statistical diagrammatic evaluation was not possible due to the low number of grains. The most numerous were the $\mathrm{S}_{7}-\mathrm{S}_{8}$ (Fig. 18A, B) and less frequently $\mathrm{S}_{12}, \mathrm{~S}_{17}$ and $\mathrm{P}_{4}$ 


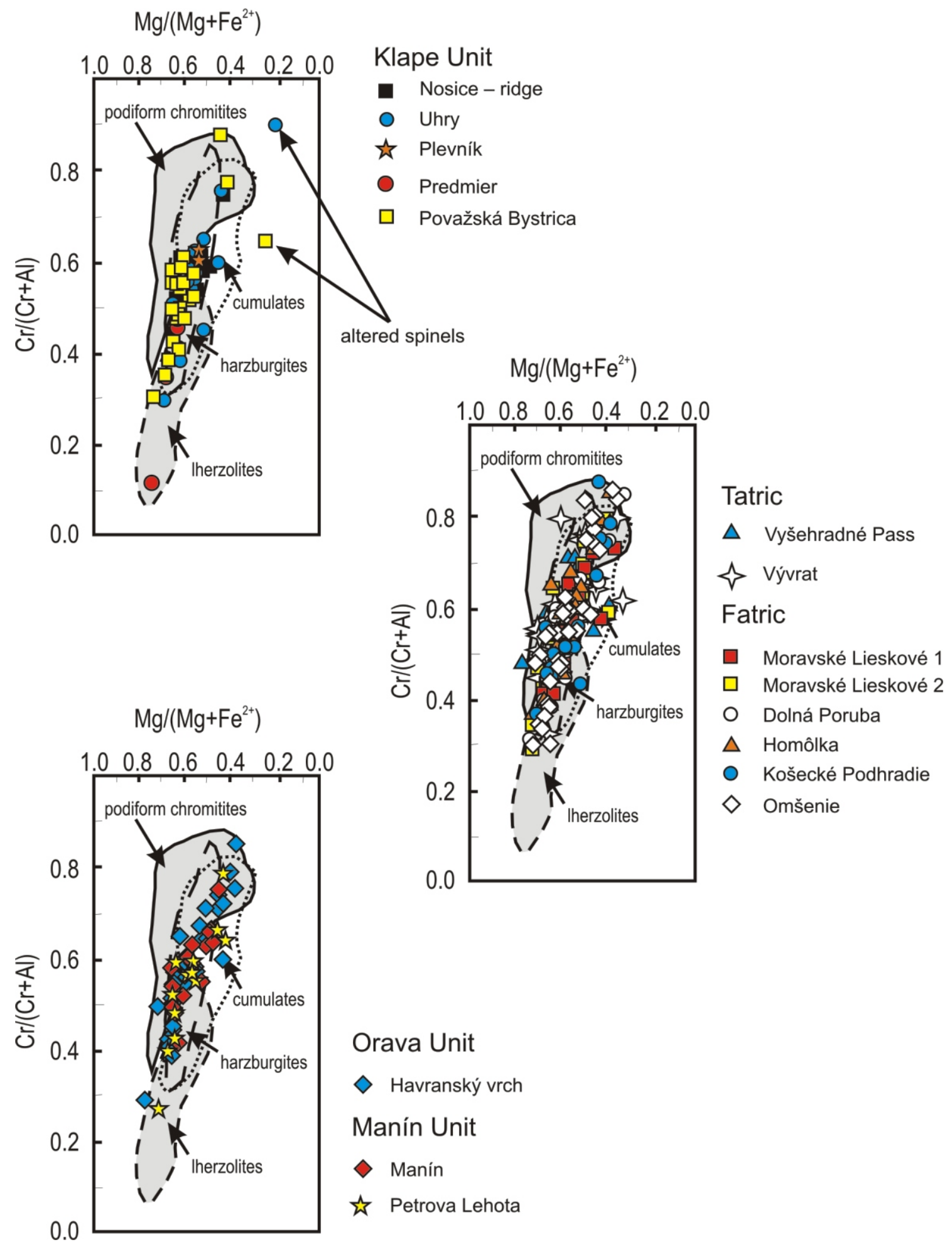

Fig. 8. The spinels measured from the individual units plotted in the $\mathrm{Mg} / \mathrm{Mg}+\mathrm{Fe}^{2+} \mathrm{vs}$. $\mathrm{Cr} / \mathrm{Cr}+\mathrm{Al}$ diagrams with fields distinguished by Pober and Faupl (1988)

Diagram fields: solid line - podiform chromitites, dotted line - cumulates, dashed line (short dashes) - Iherzolites, dashed line (long dashes) - harzburgites 


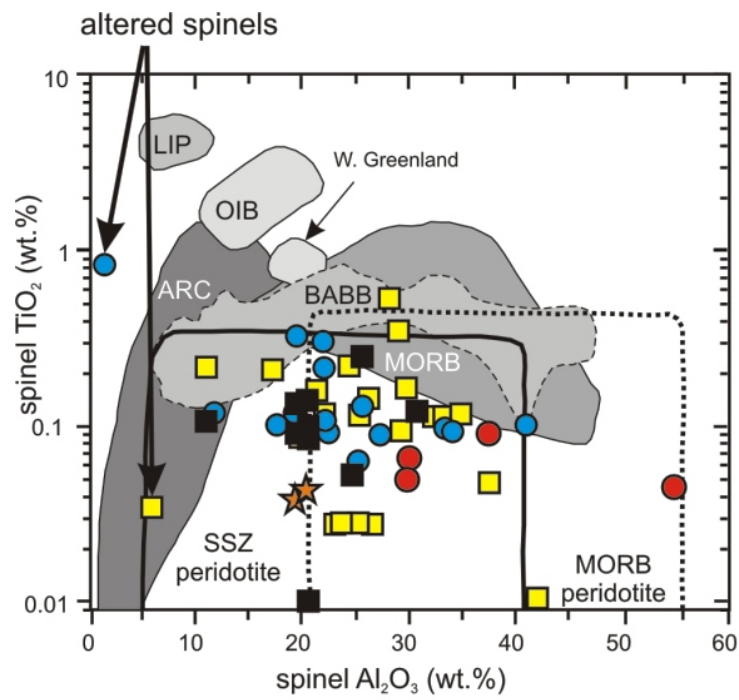

\section{Klape Unit}

- Nosice - ridge

○ Uhry

设 Plevník

Predmier

$\square$ Považská Bystrica

Tatric

$\Delta$ Vyšehradné Pass

$\leadsto$ vývrat

Fatric

$\square$ Moravské Lieskové 1

$\square \quad$ Moravské Lieskové 2

○ Dolná Poruba

$\triangle$ Homôlka

○ Košecké Podhradie

$\diamond$ Omšenie
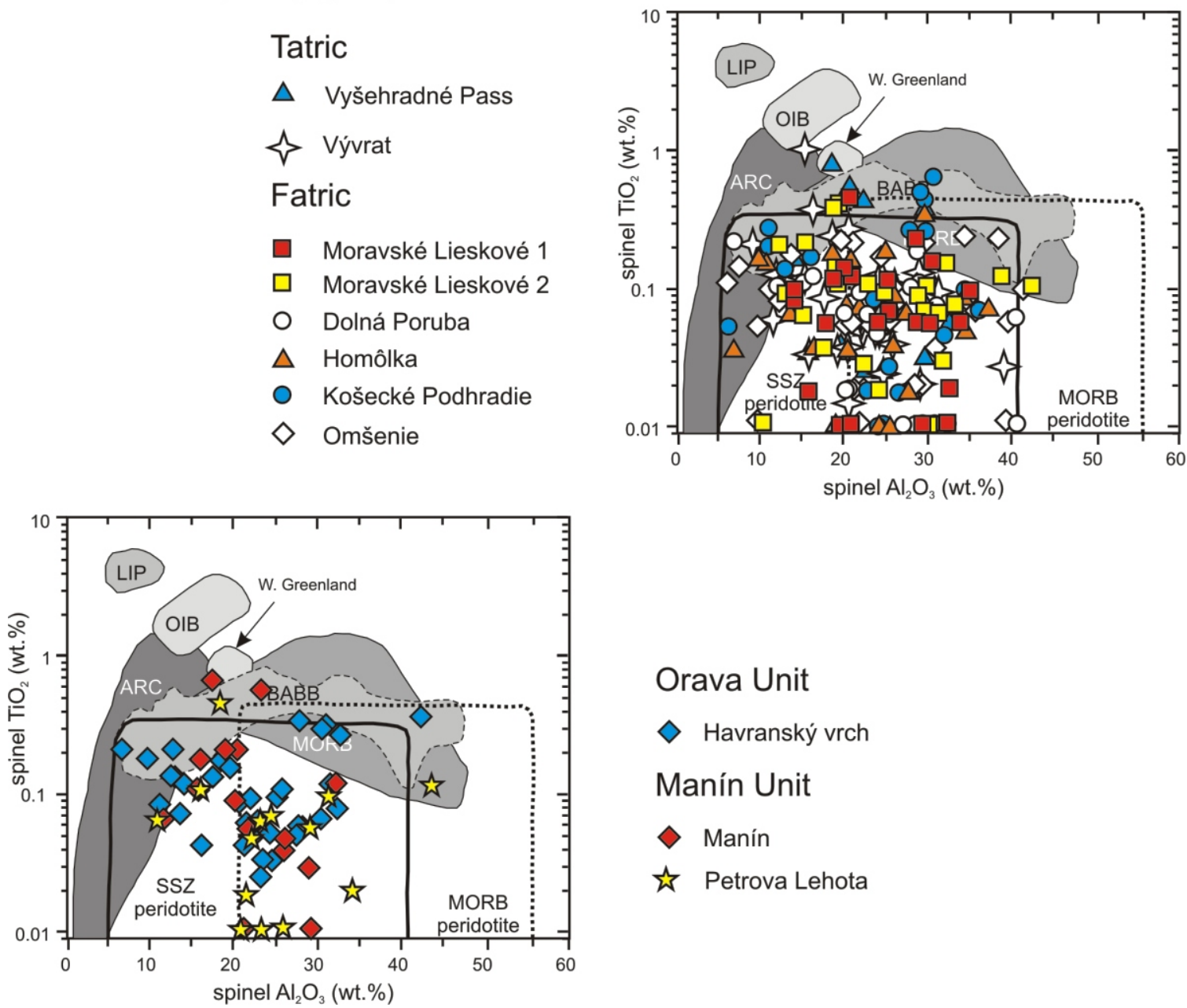

Orava Unit

Havranský vrch

\section{Manín Unit}

$\diamond$ Manín

‘ Petrova Lehota

Fig. 9. The spinels measured from the individual units plotted in the $\mathrm{TiO}_{2}$ vs. $\mathrm{Al}_{2} \mathrm{O}_{3}$ diagram of Lenaz et al. (2000) and Kamenetsky et al. (2001)

ARC - island-arc magmas, BABB - back-arc basin basalts, LIP - large igneous provinces, MORB - mid ocean ridge basalts, OIB - ocean island basalts, SSZ - supra-subduction zone peridotites 

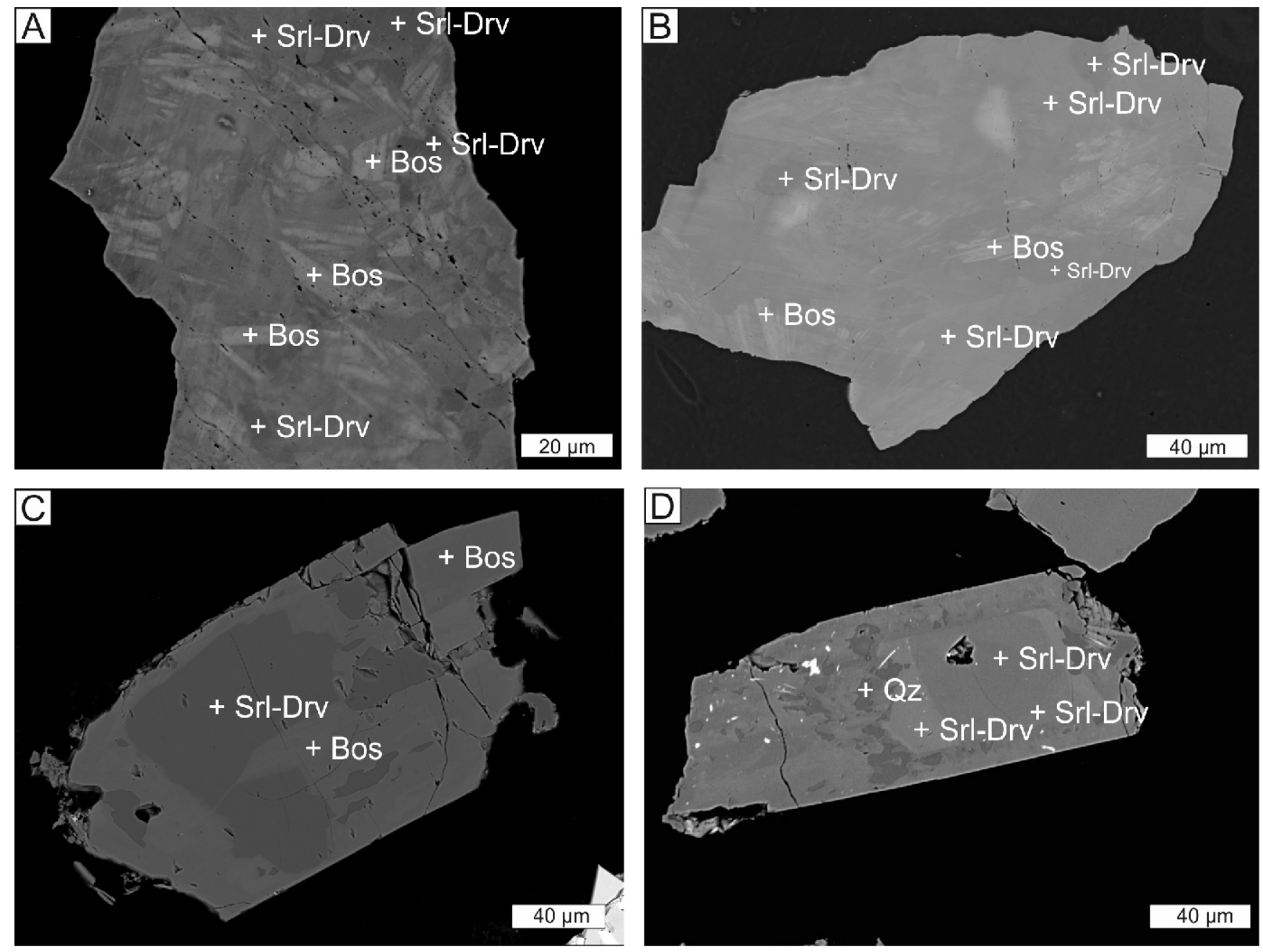

Fig. 10. BSE images of the tourmaline grains analysed, with complex-zoned fabric

A-C - complex-zoned fabric consisting of various phases of tourmaline represented by laths and irregular zones of schorl-dravitic tourmaline (Srl-Drv - schorl-dravite) and bosiite (Bos), A, B - sample N4, C - sample VD1 (normally zoned core, overgrown with complex-zoned fabric); D - complex-zoned fabric consisting of intergrown schorl-dravitic and quartz (Q) phases, sample ML2; abbreviations of mineral names from Whitney and Evans (2010)

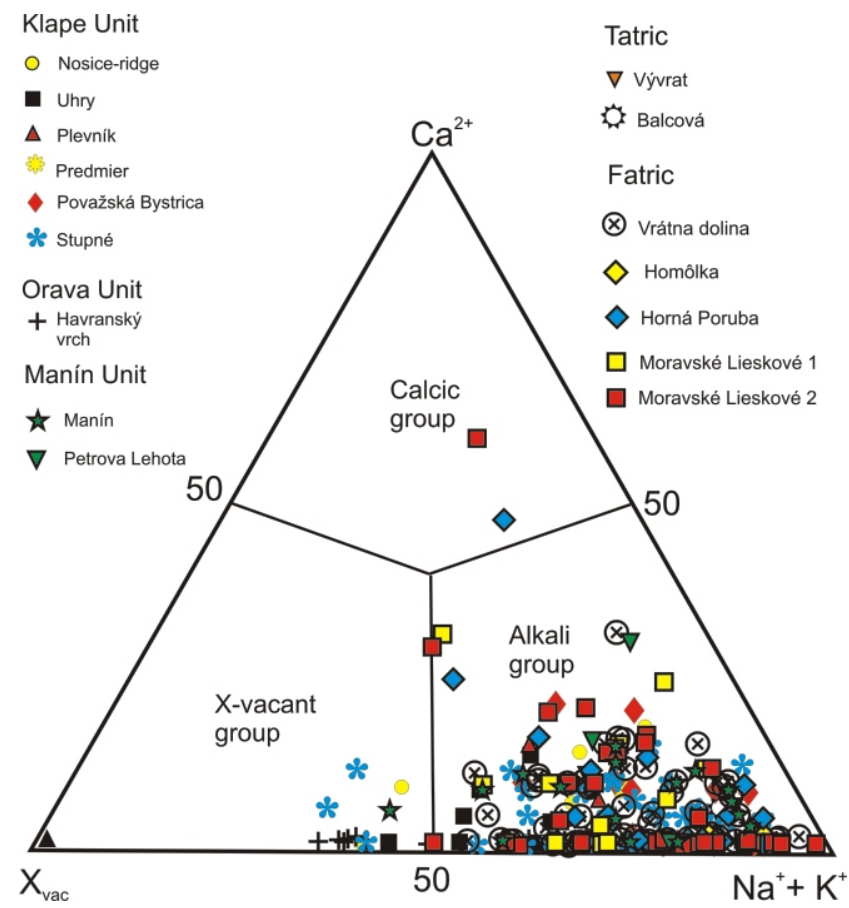

Fig. 11. Classification diagram of the tourmalines measured showing the relationship of $\mathrm{X}$-site vacancy vs. $\mathrm{Na}^{+}+\mathrm{K}^{+}$vs. $\mathrm{Ca}^{2+}$ (Henry et al., 2011) 


$\begin{array}{ll}\text { Klape Unit } & \text { Tatric } \\ 0 \text { Nosice-ridge } & \nabla \text { Vývrat } \\ \square \text { Uhry } & \text { Balcová } \\ \Delta \text { Plevník } & \text { Fatric } \\ \forall \text { Predmier } & \otimes \text { Vrátna dolina } \\ \text { \& Stupné } & \diamond \text { Homôlka } \\ \text { Orava Unit } & \diamond \text { Horná Poruba } \\ + \text { Havranský vrch } & \square \text { Moravské Lieskové 1 } \\ \text { Manín Unit } & \square \text { Moravské Lieskové 2 } \\ \text { † Manín } & \\ \nabla \text { Petrova Lehota } & \end{array}$

$\mathrm{X}$ site $=\mathrm{Na}+\mathrm{K}$ or 品 dominant; $(\mathrm{Mg}+\mathrm{Fe}) /(\mathrm{Mg}+\mathrm{Fe}+\mathrm{Li})>0.5$ $\mathrm{W}$ site = occupancy unknown

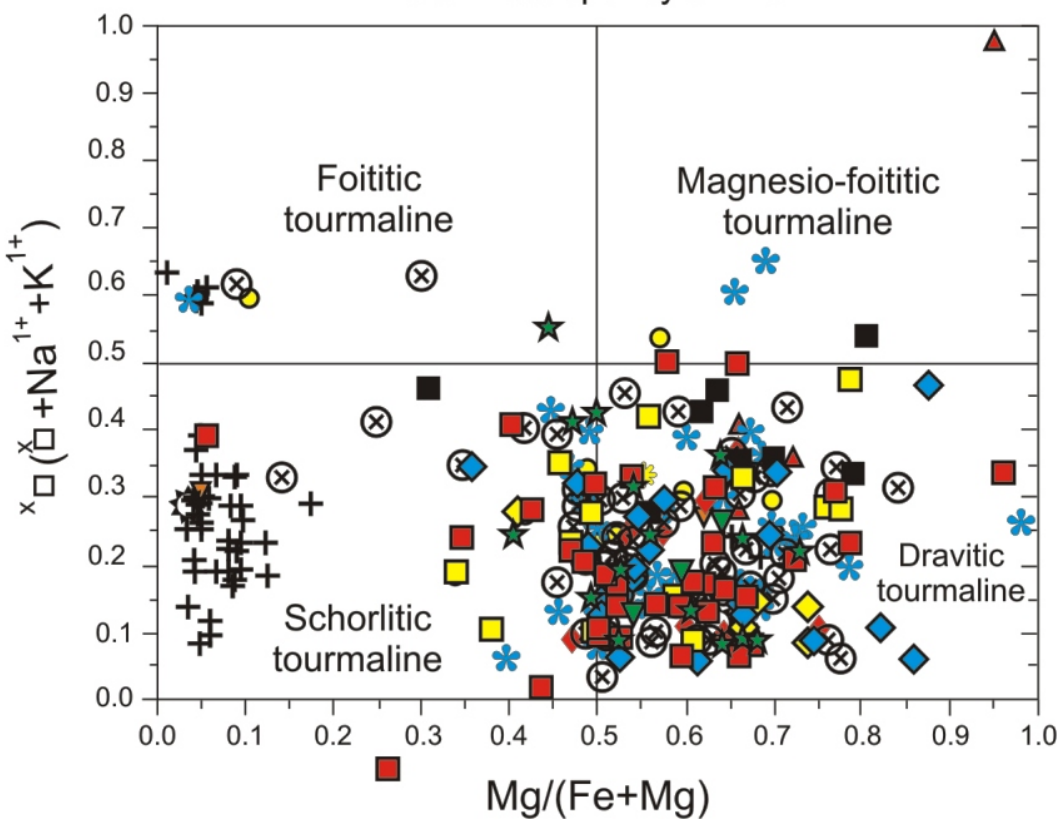

Fig. 12. $\mathrm{Fe} /(\mathrm{Fe}+\mathrm{Mg})$ vs. ${ }^{\mathrm{x}} \square /\left(\mathrm{x}_{\square}+\mathrm{Na}^{1+}+\mathrm{K}^{1+}\right)$ classification diagram of the tourmalines measured (Henry et al., 2011)

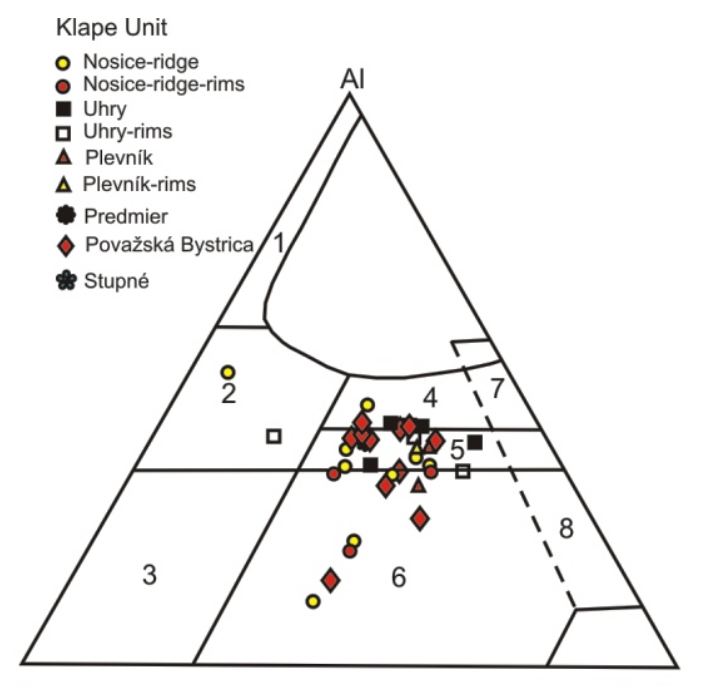

$\mathrm{Al}_{50} \mathrm{Fe}_{50}$

$\circ$

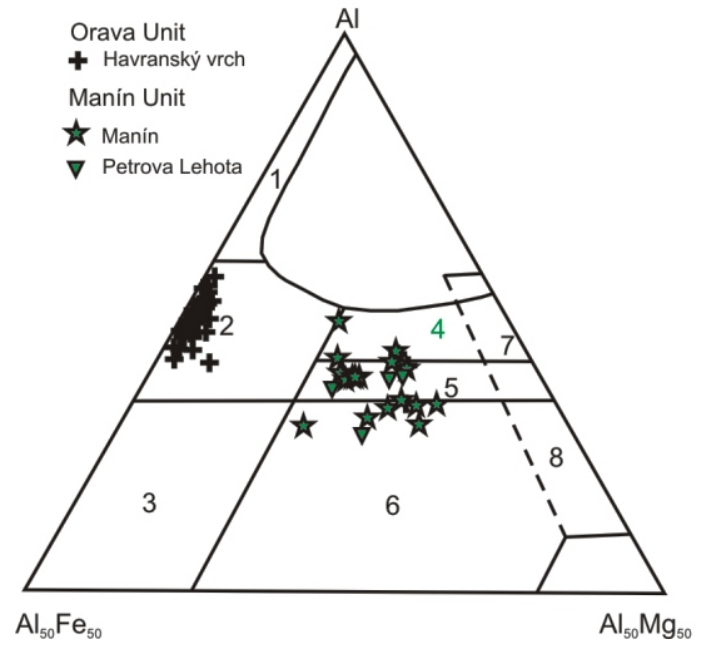

Tatric

$\boldsymbol{\nabla}$ Vývrat

Balcová

Fatric

® Vrátna dolina

$\diamond$ Homôlka

$\checkmark$ Horná Poruba

口 Moravské Lieskové 1

口 Moravské Lieskové

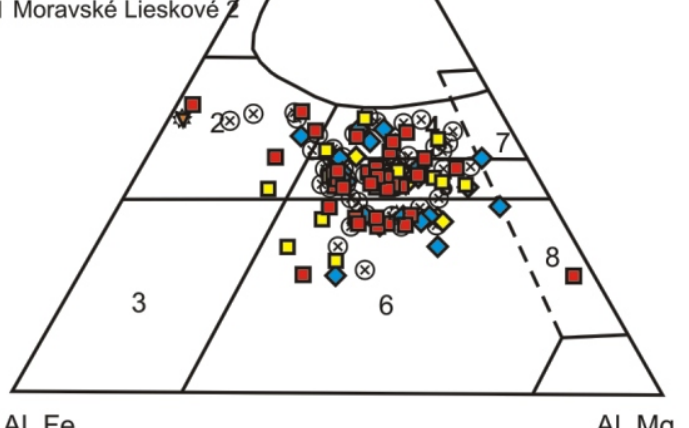

$\mathrm{Al}_{50} \mathrm{Fe}_{50}$

$\mathrm{Al}_{50} \mathrm{Mg}_{50}$

Fig. 13. Ternary diagrams exhibiting $\mathrm{Al}, \mathrm{Fe}$ and $\mathrm{Mg}$ molecular proportions of the tourmalines analysed from the individual units (from Henry and Guidotti, 1985)

Explanations of the diagram fields: 1 - Li-rich granitoid pegmatites and aplites; 2 - Li-poor granitoids and their associated pegmatites and aplites; $3-\mathrm{Fe}^{3+}$-rich quartz-tourmaline rocks (hydrothermally altered granites); 4 - metapelites and metapsammites coexisting with an Al-saturating phase; 5 - metapelites and metapsammites not coexisting with an Al-saturating phase; $6-\mathrm{Fe}^{3+}$-rich quartz-tourmaline rocks, calc-silicate rocks and metapelites; 7 - low-Ca metaultramafics and $\mathrm{Cr}$, V-rich metasedimentary rocks; 8 metacarbonates and meta-pyroxenites 


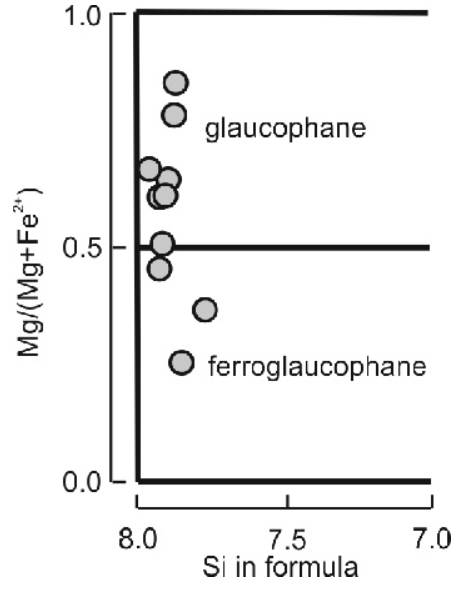

Fig. 14. Si vs. $\mathrm{Mg} /\left(\mathrm{Mg}+\mathrm{Fe}^{2+}\right)$ classification diagram of the blue amphiboles analysed from the Predmier locality (Leake et al., 1997)

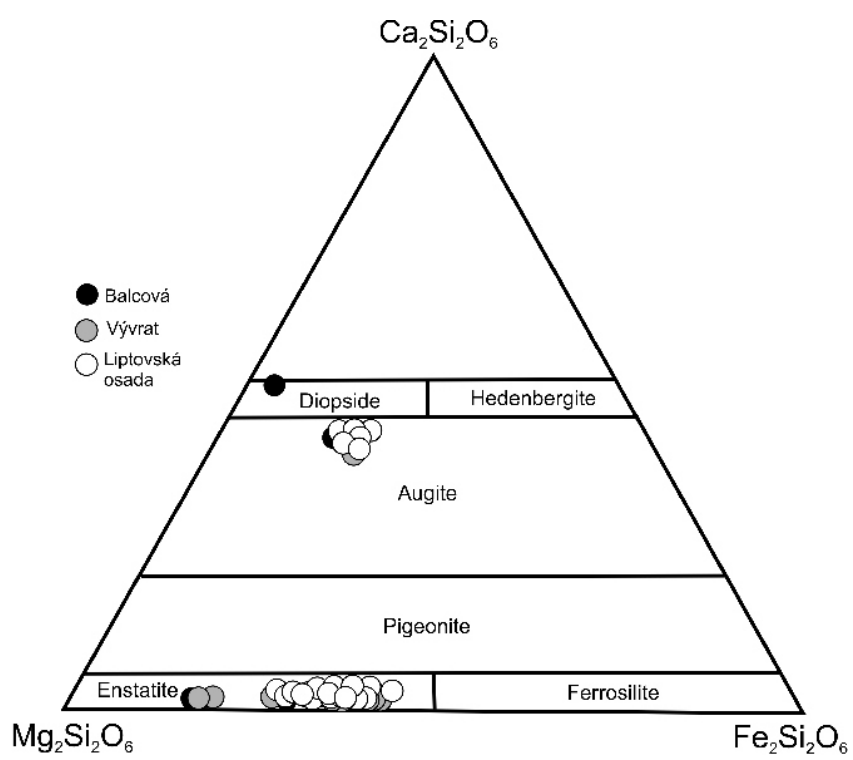

Fig. 16. Classification diagram of the pyroxenes analysed (Morimoto et al., 1988)
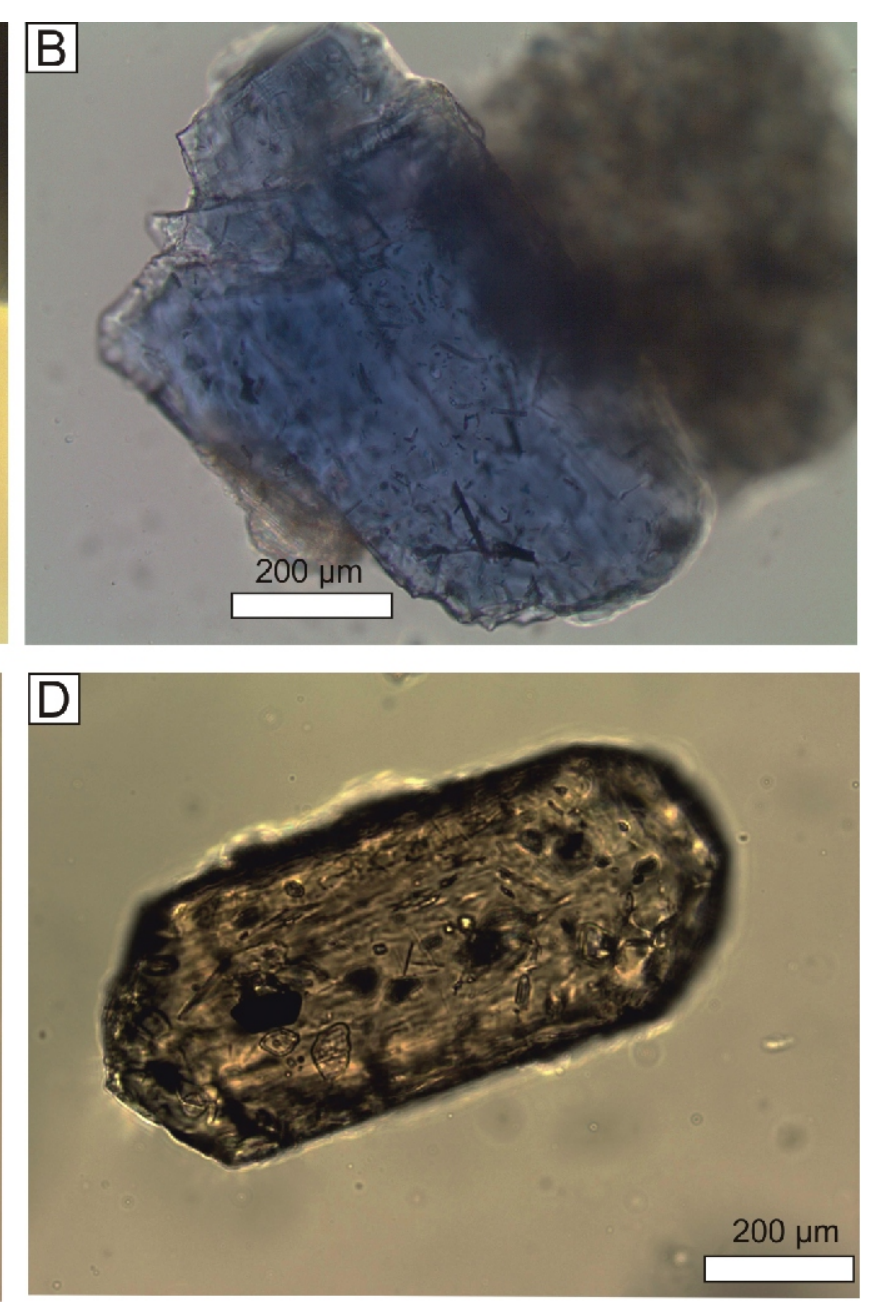
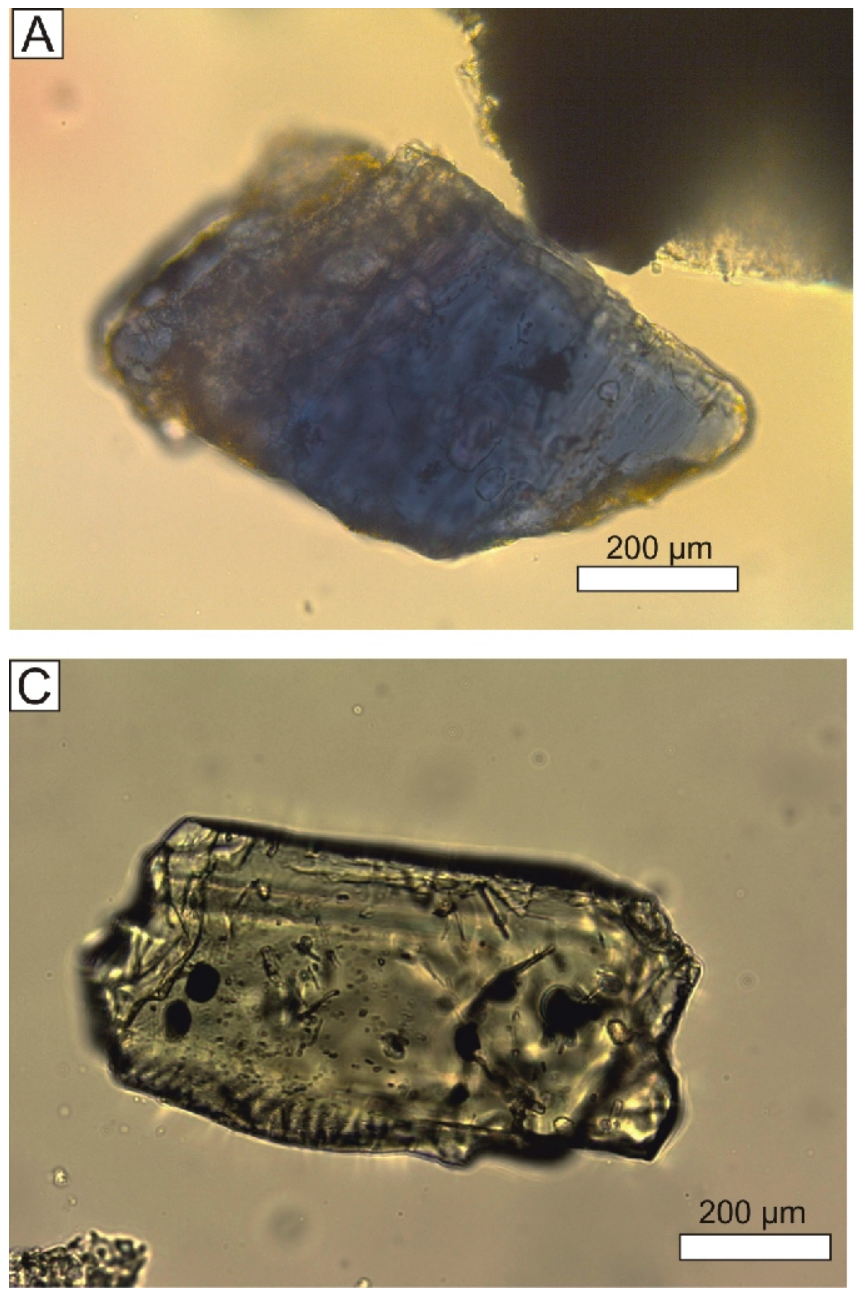

Fig. 15A, B - microphotos of blue amphiboles, Predmier locality; C, D - microphotos of pyroxenes, Balcová locality, plane polarized light 


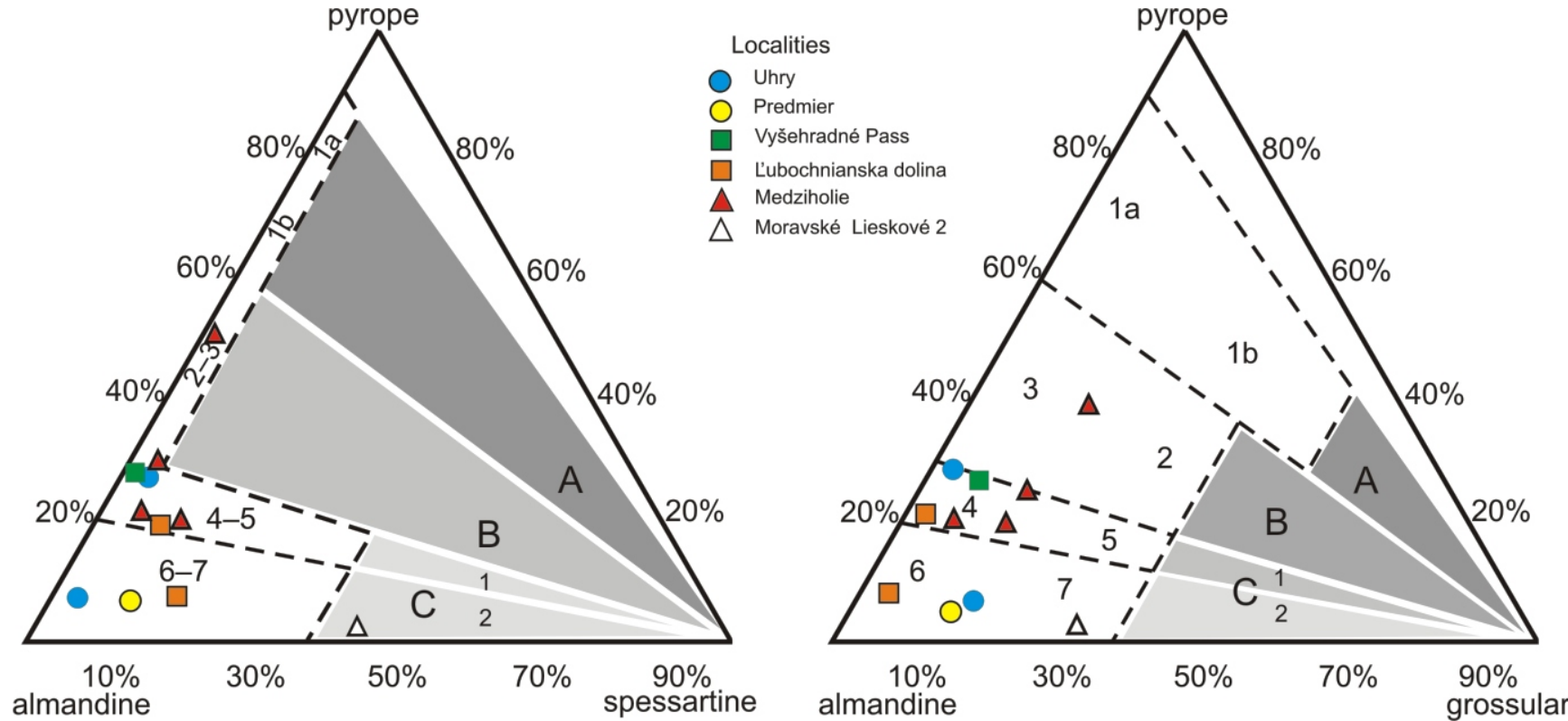

Fig. 17. Plot of the measured garnet (Grt) composition in the pyrope-almandine-grossular and pyrope-almandine-spessartine classification diagrams by Méres (2008)

Sector A. White field - garnets from UHP/HP conditions. Position around No. 1a - Grt derived from UHP eclogites, garnet peridotites and kimberlites. Position around No. 1b-Grt derived from UHP eclogites. Sector B. White field - garnets from eclogite and granulite facies conditions. Position around No. 2 - Grt derived from HP eclogites and HP mafic granulites. Position around No. 3 - Grt derived from HP felsic and intermediate granulites. Sector C. White field - garnets from amphibolite facies conditions: Sector C1 - transitional subgroup between granulite and high amphibolite facies conditions. Position around No. 4-Grt derived from gneisses metamorphosed under P-T transitional to granulite and amphibolite facies conditions. Position around No. 5 - Grt derived from amphibolites metamorphosed under transitional P-T granulite to amphibolite facies conditions. Sector C2 - subgroup amphibolite facies conditions. Position around No. $6-\mathrm{Grt}$ derived from gneisses metamorphosed under amphibolite facies conditions. Position around No. 7 - Grt derived from amphibolites metamorphosed under amphibolite facies conditions. In the C2 subgroup Grt from many other sources integrate, e.g. Grt from igneous rocks (granitoids, syenites), Grt from HP/LT metamorphic rocks, Grt from contact-metamorphosed rocks. Grey fields - immiscibility gap of Grt end-member compositions: A - from UHP/HP conditions, B - from eclogite and granulite facies conditions, $\mathrm{C}-$ from amphibolite facies conditions

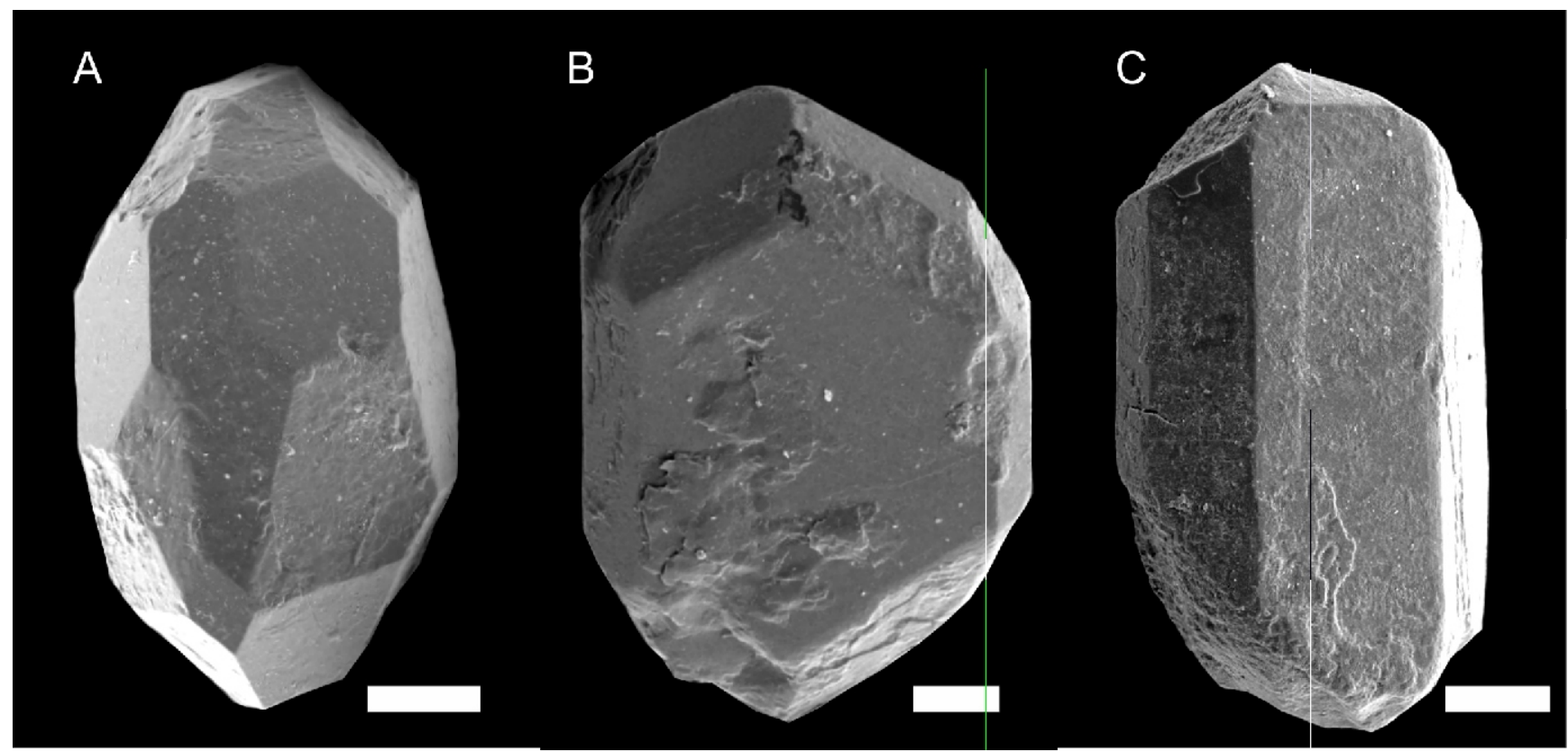

Fig. 18. Representatives of the zircon morphological types

A - Havranský vrch locality, $\mathrm{S}_{7}$; B - Moravské Lieskové 1 locality, $\mathrm{S}_{8}$; C - Stupné locality, $\mathrm{P}_{4}$; the scale bars represent $0.25 \mu \mathrm{m}$ 
(Fig. 18C) zircon subtypes. Only single grains of $\mathrm{S}_{1-3}, \mathrm{~S}_{8}, \mathrm{~S}_{18}$, $\mathrm{S}_{21-22}$ and $\mathrm{S}_{24}$ subtypes were registered.

The zircon subtypes $S_{7}-S_{8}$ of Pupin (1980) are typical of the Tatric and Veporic Variscan granites (Broska and Uher, 1991; Broska et al., 2012), whereas P zircon subtypes are typical of the late to post-Variscan, mainly A-type granites and acid volcanics of Permian age in the Western Carpathians (Broska et al., 1993, 2012; Uher and Broska, 1996). P-types of zircon are characteristic of the exotic Upohlav type of granite known from the pebble material of the Klape Unit (Uher and Marschalko, 1993). However, they are dominated by the $P_{5}$ subtype, whereas the $\mathrm{P}_{4}$ subtype were present in our samples in minor amounts.

Zircon typology under SEM reveals only the latest stages of crystallization of the individual grains. Their complete history in the form of zonation can be revealed only by transmitted light microscopy or by electron microscopy of polished grains.

\section{DISCUSSION}

Our study provides an augmented set of heavy mineral data from the Albian-Cenomanian sedimentary rocks in the Western Carpathians, representing the earliest massive input of ophiolitic detritus related to the convergence and closure of oceanic realms during the Alpine orogeny. Herein, we emphasize the new results which add to the discussion of Bellová et al. (2018).

\section{PETROGRAPHIC COMPOSITION OF THE SANDSTONES ANALYASED}

Petrographic analysis showed that the samples are mostly calcareous sandstones. The ratio of the non-calcareous components ranks them among litharenites and sublitharenites. According to the provenance diagram of Dickinson (1985), their source area can be classified as recycled orogen. These results, which show a generally immature character of the sediment, may be related to a relatively fast emergence-redeposition process and correspond well with the pebble analyses, since they show a great predominance of carbonates and other relatively unstable components (Mišík and Sýkora, 1981; Mišík et al., 1981). Moreover, the petrography results fit perfectly into the analogous, coeval, exotics-bearing deposits from the Eastern Alps (Von Eynatten and Gaupp, 1999).

\section{PERCENTAGES OF HEAVY MINERAL ASSEMBLAGES}

The heavy mineral data complemented by the ten new localities underlined the variability of the percentage ratios of heavy minerals, but with a consistent dominance of chrome-spinels, zircon, tourmaline, apatite and rutile. Other minerals, such as garnet, titanite, kyanite, monazite and epidote occur in lesser amounts, except for some local increased proportions of garnets, pyroxenes, apatite, kyanite or blue amphibole. Bellová et al. (2018) presented an erroneous interpretation of the previous data, where they indicated a seeming inconsistency in tourmaline/zircon ratio in the data from Mišík et al. (1980), Jablonský (1986) and Jablonský et al. (2001). After a later revision, a clear consistency was recognized instead. However, these previous data cannot be fully compared with those presented herein for several reasons. First, the above-cited authors used only a half-size grain fraction for analysis. Our data almost completely encompasses the average grain-size of the sandstones examined, thus most closely approximating the true content values. Second, the pre- vious authors counted the opaque minerals as well, which vastly prevail in the heavy fraction, and ratios of the translucent minerals cannot be calculated from their data. Third, Mišík et al. (1980), Jablonský (1986) and Jablonský et al. (2001) counted the Cr-spinels exclusively in transmitted light, thereby omitting a large portion of the grains. Our counting under the binocular lens provides a more realistic ratio of the $\mathrm{Cr}$-spinels vs. other minerals.

Statistical linear regression analysis of the heavy mineral pairs provided new data and showed that there is a moderate positive correlation among the ultrastable minerals of zircon, tourmaline and rutile. This confirmed the interpretation by Bellová et al. (2018) that an important source of the clastic sediment was most likely derived from older deposits from which the ultrastable minerals were reworked. This is also indicated by the very low content of euhedral zircon grains, that made it impossible to make a zircon grain typology. Although the pebble analysis showed the presence of rhyolitic and granitic pebbles (Krivý, 1969; Kamenický et al., 1974; Kamenický and Král', 1979; Mišík and Sýkora, 1981, Uher and Marschalko, 1993) from which primary zircons might have been derived, the zircon grains reworked from older strata obviously form a much larger part.

The second main source is represented by the ophiolitic input characterized by spinels (mainly Cr-spinels), which are locally accompanied by pyroxenes and blue amphiboles. Grains of complexly zoned tourmaline most likely belong to this assemblage (Aubrecht et al., 2020), which: (1) did not appear in the previous detrital successions of the Western Carpathians (Aubrecht, 1993, 1994, 2001; Aubrecht and Krištín, 1995; Aubrecht et al., 2017), (2) occurs in HP to UHP rocks accompanying ophiolite complexes (Altherr et al., 2004; Marschall et al., 2006, 2008; Miller et al., 2009; Konzett et al., 2012; Broska et al., 2015; Plašienka et al., 2019), (3) is most likely the portion of tourmaline which causes the moderately positive correlation between tourmaline and $\mathrm{Cr}$-spinels. Scarcer minerals, such as garnet, kyanite, staurolite and sillimanite also reflect some small and uneven presence of continental-crust metamorphic rocks (reflected also by a strong positive correlation between garnet and staurolite). Staurolite, sillimanite and kyanite indicate the presence of rocks metamorphosed in various $\mathrm{P} / \mathrm{T}$ conditions, but point mostly to the medium- to high-grade barrovian metamorphism. Heavy mineral percentages provided herein are also highly consistent with the results from the Arosa and Walsertal zones below the Austroalpine thrust (Winkler and Bernouilli, 1986; Winkler, 1988), as well as from the Tannheim, Losenstein and Branderfleck formations of the lowest Upper Austroalpine nappes in the Eastern Alps (Von Eynatten and Gaupp, 1999; Wagreich, 2003). There is a slight difference in the contents of blue amphiboles, which occur in the Alpine units in relatively small, but constant amounts, which are higher in the Branderfleck Formation (Von Eynatten and Gaupp, 1999). The content of chloritoid grains was also constant, part of which was related to the HP/LT blueschist-facies metamorphism (Von Eynatten and Gaupp, 1999). In the samples presented herein, chloritoid was not analysed.

\section{COMPARISON OF HEAVY MINERAL DATA AND PEBBLE ANALYSIS}

The constraints provided by heavy mineral analysis are largely consistent with the previously published results of pebble analysis (Mišík and Sýkora, 1981; Mišík et al., 1981). Rocks composing the pebble material are dominated by carbonates (limestones and dolomites). However, they are mostly irrelevant for comparison of the siliciclastic material. Pebbles of siliciclastic rocks (sandstones and quartzites) are usually 
among the most common relevant rocks, and this corresponds well with the main presumed source - older siliciclastic successions. However, the possible ophiolitic source left little information in the gravel fraction. Pebbles of basaltic volcanic rocks occur commonly, whereas glaucophanite pebbles (Ivan and Sýkora, 1993; Ivan et al., 2006), gabbros (Ivan et al., 1996) and tourmalinites with complex-zoned tourmaline (Bačík et al. 2008) are relatively rare. Despite some mentions of serpentinite detritus in the sand fraction (Mišík and Sýkora, 1981; Mišík et al., 1981), only one serpentinite pebble has been found so far (P. Ivan - personal communication, 2019). This can be interpreted in terms of the very low resistance of serpentinized ultrabasic rocks to weathering and transport. Although the pebble material and modal analysis of the sandstones show low sediment maturity, this is not valid for the heavy minerals. The high ZTR index, complemented by very stable Cr-spinels, indicate a very small portion of unstable minerals. Unprotected by surrounding rock in pebbles, minerals such as amphiboles, olivine and pyroxenes have been subject to severe intrastratal dissolution, which also distorted the real picture that could have been gained from the heavy mineral analysis.

Ophiolitic pebble material is strongly underrepresented with respect to the vast amount of $\mathrm{Cr}$-spinels (and other potential ophiolitic minerals) in the sand grade material. On the other hand, the stability of limestones as pebbles is also very low, but these are much more common. Consequently, the most probable interpretation is that the ophiolites were resedimented from older, Upper Jurassic ophiolitic mélanges and olistostromes, whereas the limestones were derived from new nappes and slices that emerged during the Albian. Alternatively, the ophiolitic detritus was derived from more distant sources and underwent longer transport.

\section{PALAEOGEOGRAPHIC CONSTRAINTS}

The first research concerning Western Carpathian Cretaceous exotic detritus was carried out by Matějka and Andrusov (1931), Zoubek (1931) and Andrusov (1938), who investigated the "Upohlav" conglomerates in the Pieniny Klippen Belt. Their source was interpreted to be an exotic Pieniny Ridge (Andrusov, 1938, 1945), which was later renamed by Birkenmajer (1988) as the Andrusov Ridge. According to Birkenmajer (1977, 1988), the Andrusov Ridge was positioned south of the Kysuca-Pieniny Basin (passing into oceanic crust) and north of the Central Western Carpathians. According to Marschalko (1986), the transport direction of the exotic material in the Klape Unit was from the south and south-east. This would indicate the position of the Klape Unit north of the Andrusov Ridge. This opinion was challenged by Birkenmajer (1988), who placed this unit south of the ridge. The Andrusov Ridge was inferred to be an accretionary wedge formed by subduction of the Jurassic, Penninic-Vahic Ocean (e.g., Mahel', 1981, 1989; Birkenmajer, 1988).

In the Tatric and Fatric Poruba Formation, the transport directions largely differ from those in the Klape Unit (Jablonský, 1978, 1986). The data in the Tatric units are largely scattered, but in general, trough-parallel transport dominated, with some lateral transport directions coming from the south (in the Nízke Tatry Mts.). In the Fatric units (Krížna Nappe), there were both southern and northern sources indicated by the measurements. Hence, Mišík et al. (1980) suggested the presence of two additional exotic sources, the Ultratatric and Ultrakrižna ridges, which made the palaeogeographic situation quite complicated and did not reflect the relative uniformity of the clastic material.
Pebbles of basaltic volcanics of Late Jurassic-Early Cretaceous K-Ar age (Rybár and Kantor, 1978; Birkenmajer and Pécskay, 2000) would fit into an attribution to Penninic ophiolites. Some exotic clasts were interpreted as being derived from the Carpathian foreland, e.g. Namurian black coal (Havlena, 1956; Šilar, 1956) or from non-metamorphosed Devonian limestone (Tomaś et al., 2004).

However, there are many exotic clasts which seem to be derived from more southern zones representing the Inner Western Carpathians and even Dinarides. There are pebbles to blocks of southern types of Triassic, e.g. Wetterstein-type, platform limestones typical of the Silicic units; exotic granitic pebbles (the "Upohlav-type") are most similar to those of A-type granites, e.g. Turčok or Velence Massif (Uher and Marschalko, 1993; Uher et al., 1994; Uher and Pushkarev, 1994; Uher and Broska, 1996). The Devonian limestone pebbles noted above might be alternatively derived from a more southern source, such as the Transdanubian Central Range. Deep-water to oceanic Triassic deposits are very characteristic, indicating their relationship with the Meliata Ocean (Mišík et al., 1977; Birkenmajer et al., 1990). The Triassic deep-sea deposits found among the pebble material of the exotic clasts (deep-water limestones and cherts) are even older (Lower Anisian) than those found in the Meliata Unit. Moreover, radiometric dating of some glaucophanite pebbles indicated a Jurassic age of metamorphism, which is in accordance with the closure of the Meliata ocean of the Neotethys (Rybár and Kantor, 1978; Dal Piaz et al., 1995). A dominance of Neotethyan elements in the exotic conglomerates led to speculations about the proximity of the Meliata and Oravic domains (e.g., Mišík, 1978; Mišík and Sýkora, 1981), although later some authors favoured an alternative explanation of two different Triassic troughs, south and north of the Central Western Carpathians (Birkenmajer et al., 1990).

In the Eastern Alps, where the situation regarding the exotic material is very similar to that in the Western Carpathians, there has also been a long-lasting debate about the northern (Penninic) and southern (Meliata-Vardar) sources of the ophiolite detritus in Cretaceous deposits (e.g., Decker et al., 1987; Pober and Faupl, 1988; Faupl and Pober, 1991; Faupl and Wagreich,1992; Wagreich et al., 1995; Von Eynatten and Gaupp, 1999; Wagreich, 2003). In recent times, however, a southern source from a propagating Jurassic accretionary wedge, which contained obducted dismembered ophiolites, has become the consensus among researchers (e.g., Stern and Wagreich, 2013; Krische et al., 2014; Gawlick et al., 2015).

In the Western Carpathians, in an attempt to unify both ophiolitic occurrences, with primary in the south and secondary (redeposited) in the north, Plašienka $(1995,1996)$ made the radical suggestion that the Klape Unit belongs to the Fatric domain, and its exotic flysch deposits represent merely a proximal part of the Poruba Formation turbiditic fan (the "Fatric Hypothesis" further in the text). There are, however, many counter-arguments against this Fatric Hypothesis (Mišík, 1996). Among them, the most important are the diminishing size of the Poruba Formation clasts southwards, as well as the large volume of Coniacian-Santonian exotics in the Klape and Kysuca units of the Pieniny Klippen Belt with identical material, which had to originate exclusively by redeposition from Albian-Cenomanian sediments, that is, if the Fatric Hypothesis was true. Although there are some signs of cannibalism of earlier exotic material during the Senonian (clasts of the Cenomanian Orlové sandstones and of Albian conglomerates in the Coniacian-Santonian deposits, etc. - Mišík and Sýkora, 1981; Marschalko, 1986), the diversity of pebble material appears not to be af- 


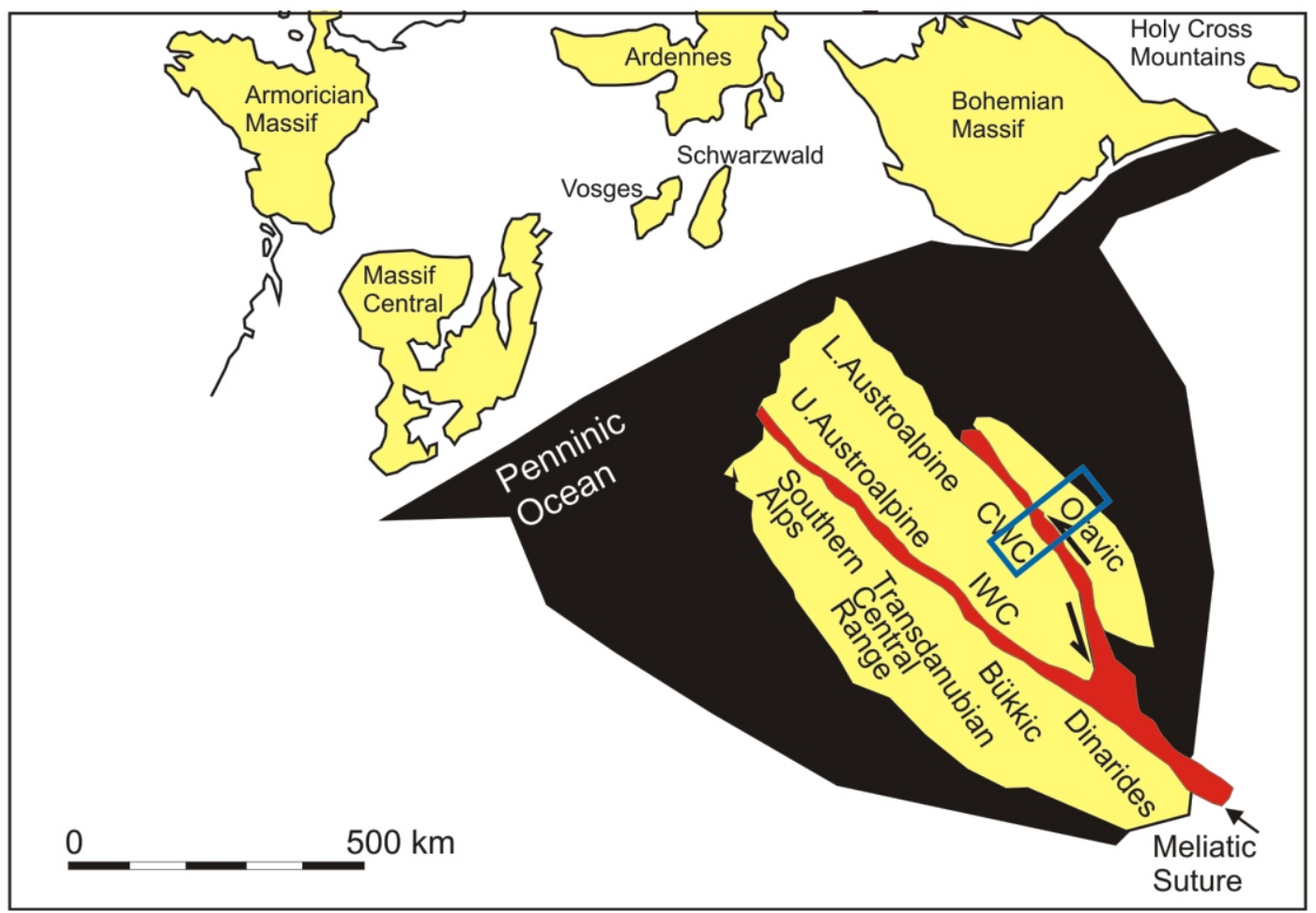

Fig. 19. Simplified (limited number of microplates) schematic palaeogeographic map of the Alpine-Carpathian units (Albian) in the context of the possible position of the source of exotic ophiolitic material

fected by any resedimentation, and is even higher than in the Albian with a higher portion of the least stable carbonate pebbles (Marschalko, 1986). The final, most significant counter-argument against the Fatric Hypothesis was the discovery of exotic ophiolitic detritus in the Upper Aptian-Albian strata of the Czorsztyn Unit of Oravicum (Aubrecht et al., 2009). This unit, which was presumably deposited on the most elevated Oravic part, the so-called Czorsztyn Ridge, was considered to be free of any exotic material (e.g., Mišík and Marschalko, 1988) and was interpreted to be situated on the other side of the Kysuce-Pieniny Basin than the Andrusov Ridge. Cr-spinels were also found in the Albian-Cenomanian Trawne Member, which is attributed to the Branisko (Kysuca) Unit (Winkler and Ślączka, 1994). These counter-arguments were not accepted by Plašienka, and the Fatric Hypothesis still persists in his palaeogeographic reconstructions of the Western Carpathians (e.g., Plašienka and Soták, 2015; Plašienka, 2018, 2019).

In our interpretation, we maintain the notion put forward by Aubrecht et al. (2009) and Bellová et al (2018) regarding the doubled suture nature of the closed Triassic (Neotethys) oceanic branch. The southern suture is represented by the Meliata suture zone and the northern one by the (Andrusov) Ridge, which was positioned between the West-Carpathian externides and internides. The northern branch was juxtaposed versus the internides by strike-slip motion in the Barremian to Albian interval and persisted there until the Paleogene (as the "Neopieninic Cordillera" - Mišík et al., 1991). Such an arrangement of the exotic source fits well with the conclusions made by Marschalko (1986), who argumented that the exotic source represented a long-lasting elevation formed in a strike-slip zone rather than a compressional wedge, which would be rapidly destroyed.
The following palaeogeographic evolution scenario is proposed (Figs. 19 and 20, see also Aubrecht et al., 2009). Middle Jurassic Penninic rifting caused detachment of the West-Carpathian internides (Austroalpine units) from their original position south of the Massif Central and the Oravic segment from its position in continuation of the Moldanubian Zone of the Bohemian Massif. The NE-SW orientation of the initial rifting corresponds with many palaeogeographic reconstructions (see discussion in Aubrecht and Túnyi, 2001). The Oravic segment was originally situated in lateral continuity with the West-Carpathian internides segments (Michalík, 1994). South of both segments, the older Triassic Meliata Ocean was situated (part of the Neotethys). This ocean closed in the Late Jurassic when the crustal segments, which were derived from the North-European Platform, collided with the South-Alpine/Dinaridic segments. Remnants of the ocean were arranged in a subduction mélange along the Meliatic suture zone. During the Cretaceous, the amalgamated blocks rotated further clockwise to their final NW-SE orientation in the Paleogene (see Túnyi and Márton, 2002; Csontos and Vörös, 2004; Kováč et al., 2016). The rotation caused detachment of the Oravic segment from its lateral position, and its relative lateral shift along the northern margin of the Central Western Carpathians (Fig. 19). The Meliatic mélange was then secondarily positioned in the zone between the Oravic segment and Central Western Carpathians, where it formed an elevated ridge (Andrusov ridge) that became the source of exotic pebbles and ophiolitic detritus. The ridge simultaneously fed the Klape, Tatric and Fatric units on the SW and the Oravic units on the NE (Fig. 20). 


\section{NE}

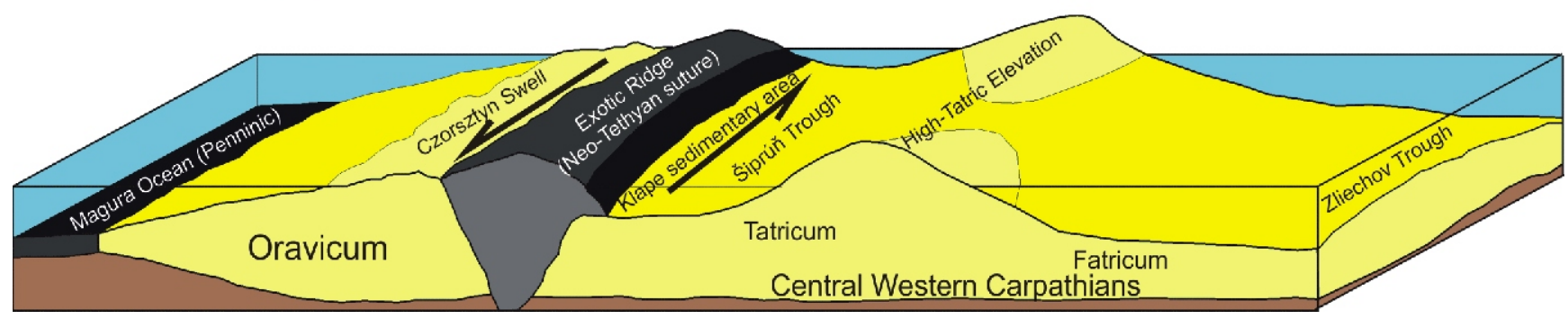

Fig. 20. Palaeogeographic block-diagram sketch showing possible position of the units examined in the Albian and the source of the ophiolitic clastics - the Exotic Ridge, from Bellová et al. (2018)

\section{CONCLUSIONS}

Sandstones from the Albian-Cenomanian exotics-bearing flysch deposits were analysed for their heavy-mineral content (Klape Unit in the Pieniny Klippen Belt, Tatric and Fatric zones of the Central Western Carpathians, Manín Unit of the Pieniny Klippen Belt and the Orava Unit of the Pieniny Klippen Belt. The results are as follows:

1. The sandstone samples analysed are fine- to medium-grained, petrographically mostly being litharenites (Klape and Orava units), with overlap on sublitharenites (Tatric units) and quartz arenites (Fatric and Manín units).

2. Most of the heavy-mineral samples were dominated by chrome-spinels, zircon, tourmaline, apatite and rutile in various ratios. Garnet is relatively rare and unevenly distributed, with local abundances of up to $78 \%$. At the Havranský vrch locality, there is a considerable kyanite content. In some samples, there were important occurrences of blue amphiboles and pyroxenes. No significant differences were observed between the individual units.

3. The heavy mineral spectra analysed indicate a large input of minerals of predominantly ophiolitic provenance, such as Cr-spinels, blue amphiboles, and complex-zoned tourmaline. Zircon, the remaining tourmaline, and rutile were likely derived from older sediments. Unevenly distributed garnet, staurolite, kyanite, and sillimanite, which occurred in relatively small amounts, were mostly derived from metamorphic rocks of the continental crust of various grade of metamorphism. The pyroxenes are not necessarily related to an ophiolitic source, but may have been derived from coeval Albian volcanic rocks.
4. The results show a close similarity with the coeval strata from the Eastern Alps and other exotics-bearing successions in the Alpine-Carpathian-Dinaridic area.

5. The results are partially inconsistent with previous pebble analyses, since pebbles derived from the ophiolitic source are quantitatively underrepresented with respect to the dominant $\mathrm{Cr}$-spinels in the sand fraction. This can be explained by lower resistance of the ophiolite rocks (mainly serpentinites), and by contrast the strong resistance of $\mathrm{Cr}$-spinels to alteration. However, the great prevalence of even less resistant carbonate pebbles indicate that the ophiolites might have been resedimented from earlier, Jurassic mélange deposits, whereas the carbonates were derived from primary nappe units in the vicinity.

6 . Based on the Jurassic ages of high-pressure metamorphism detected in the glaucophanite pebbles, we support the idea that the ophiolitic source represented a Triassic suture of the Neotethys rather than from a Jurassic Penninic one. Our palaeogeographic model is based on a tectonic secondary doubling of this suture zone, which was placed north of the Central Western Carpathians by lateral transport of the Oravic segment, thus forming a common source for exotic clasts to the Pieniny Klippen Belt and to the Central Western Carpathians.

Acknowledgements. The authors acknowledge financial support from the projects APVV 17-0170, VEGA 2/0028/17 and VEGA 1/0115/18. Suggestions and comments from Prof. M. Wagreich (Vienna University), Dr. D. Salata (Jagiellonian University, Kraków) and an anonymous reviewer greatly helped to improve this article.

\section{REFERENCES}

Altherr, T., Topuz, G., Marschall, H., Zack, T., Ludwig, T., 2004. Evolution of a tourmaline-bearing lawsonite eclogite from the Elekdağ area (Central Pontides, N Turkey): evidence for infiltration of slab-derived B-rich fluids during exhumation. Contributions to Mineralogy and Petrology, 148: 409-425.

Andrusov, D., 1938. Etude géologique de la zone des Klippes internes des Carpathes Occidentales, III partie: Tectonique. Rozpravy Státního Geologického Ústavu ČSR, 9: 1-135.

Andrusov, D., 1945. Geological research of the Inner Klippen Belt Western Carpathians. Part. IV (Stratigraphy of the Dogger and Malm), Part. V (Stratigraphy of the Cretaceous) (in Slovak). Práce Štátneho geologického Ústavu, 13: 1-176.

Aubrecht, R., 1993. Clastic admixture in Dogger crinoidal limestones of Czorsztyn Unit. Geologica Carpathica, 44: 105-111.
Aubrecht, R., 1994. Heavy mineral analyses from "Tatric" units of the Malé Karpaty Mts. (Slovakia) and their consequence for the Mesozoic paleogeography and tectonics. Mitteilungen der Österreichischen Geologischen Gesellschaft, 86: 121-132.

Aubrecht, R., 2001. Jurassic heavy mineral distribution provinces of the Western Carpathians. Mineralia Slovaca, 33: 473-486.

Aubrecht, R., Krištín, J., 1995. Provenance of detrital tourmaline in the Lower Jurassic of the Malé Karpaty Mts. Mineralia Slovaca, 27: $37-44$.

Aubrecht, R., Túnyi, I., 2001. Original orientation of neptunian dykes in the Pieniny Klippen Belt (Western Carpahtians): the first results. Contributions to Geophysics and Geodesy, 31: 557-578.

Aubrecht, R., Méres, Š., Sýkora, M., Mikuš,T., 2009. Provenance of the detrital garnets and spinels from the Albian sediments of 
the Czorstyn Unit (Pieniny Klippen Belt, Western Carpathians, Slovakia). Geologica Carpathica, 60: 463-483.

Aubrecht, R., Sýkora, M., Uher, P., Li, X.-H., Yang, Y.-H., Putiš, M., Plašienka, D., 2017. Provenance of the Lunz Formation (Carnian) in the Western Carpathians, Slovakia: heavy mineral study and in situ LA-ICP-MS U-Pb detrital zircon dating. Palaeogeography, Palaeoclimatology, Palaeoecology, 471: 233-253.

Aubrecht, R., Bačík, P., Mikuš, T., Bellová, S., 2020. Detritic tourmalines with complex zonation in the Cretaceous exotic flysches of the Western Carpathians: where did they come from? Lithos, 362-363, doi: 10.1016/j.lithos.2020.105443

Bačík, P., Uher, P., Sýkora, M., Lipka, J., 2008. Low-Al tourmalines of the schorl-dravite-povondraite series in redeposited tourmalinites from the Western Carpathians, Slovakia. Canadian Mineralogist, 40: 1117-1129.

Bellová, S., Aubrecht, R., Mikuš, T., 2018. First results of systematic provenance analysis of the heavy mineral assemblages from the Albian to Cenomanian exotic flysches of the Klape Unit, Tatricum, Fatricum and some adjacent units. Acta Geologica Slovaca, 10: 45-64.

Birkenmajer, K., 1977. Jurassic and Cretaceous lithostratigraphic units of the Pieniny Klippen Belt, Carpathians, Poland. Studia Geologica Polonica, 45: 1-158.

Birkenmajer, K., 1987. The Trawne Member (Upper Albian-Upper Cenomanian) - a flysch development in the Branisko Nappe, Pieniny Klippen Belt, Carpathians. Studia Geologica Polonica, 92: $29-40$.

Birkenmajer, K., 1988. Exotic Andrusov Ridge: its role in plate-tectonic evolution of the West Carpathian foldbelt. Studia Geologica Polonica, 91: 7-92.

Birkenmajer, K., Kozur, H., Mock, R., 1990. Exotic Triassic pelagic limestone pebbles from the Pieniny Klippen Belt of Poland: A further evidence for Early Mesozoic rifting in West Carpathians. Annales Societatis Geologorum Poloniae, 60: 3-44.

Birkenmajer, K., Pécskay, Z., 2000. Early Cretaceous K-Ar age of a large basalt olistolith at Biala Woda, Pieniny Klippen Belt, West Carpathians, Poland. Studia Geologica Polonica, 117: 27-35.

Bónová, K., Bóna, J., Spišiak, J., Kováčik, M., 2017. Chromian spinels from the Magura Unit (Western Carpathians, Eastern Slovakia) - their petrogenetic and paleogeographic implications. Geological Quarterly, 61 (1): 3-18.

Bónová, K., Mikuš, T., Bóna, J., 2018. Is Cr-spinel geochemistry enough for solving the provenance dilemma? Case study from the Palaeogene sandstones of the Western Carpathians (Eastern Slovakia). Minerals, 8: 543.

Broska, I., Uher, P., 1991. Regional typology of zircon and its relationship to allanite/monazite antagonism (on an example of Hercynian granitoids of Western Carpathians). Geologica Carpathica, 42: 271-277.

Broska, I., Vozár, J., Uher, P., Jakabská, K., 1993. Zircon typology from Permian rhyolites dacites and their pyroclastics (Western Carpathians). In: Geodynamický model a hlbinná stavba Západných Karpát (eds. M. Rakús and J. Vozár): 151-158. GÚDŠ, Bratislava.

Broska, I., Petrík, I., Uher, P., 2012. Accessory Minerals of the West-Carpathian Granitic Rocks (in Slovak with English summary). Veda, Bratislava.

Broska, I., Janák, M., Bačík, P., Kumar, S., 2015. Tourmaline from the eclogite hosting gneisses in the Tso Morari UHP metamorphic terrane (Ladakh, India): characteristics and evolution. Periodico di Mineralogia, ECMS 2015: 37-38.

Brown, E.H., 1977. The crossite content of Ca-amphibole as a guide to pressure of metamorphism. Journal of Petrology, 18: 53-72.

Csontos, L., Vörös, A., 2004. Mesozoic plate tectonic reconstruction of the Carpathian region. Palaeogeography, Palaeoclimatology, Palaeoecology, 210: 1-56.

Dal Piaz, G. V., Martin, S., Villa, I., Gosso, G., Marschalko, R. 1995. Late Jurassic blueschist facies pebbles from the Western
Carpathian orogenic wedge and paleostructural implications for Western Tethys evolution. Tectonics, 14: 874-885.

Decker, K., Faupl, P., Müller, A., 1987. Synorogenic sedimentation on the Northern Calcareous Alps during the Early Cretaceous. In: Geodynamics of the Eastern Alps (eds. H.W. Flügel and P. Faupl): 126-141. Deuticke, Wien.

Dick, H.J.B., Bullen, T., 1984. Chromian spinel as a petrogenetic indicator in abyssal and alpine-type peridotites and spatially associated lavas. Contributions to Mineralogy and Petrology, 86: 54-76.

Dickinson, W.R., 1985. Interpreting provenance relations from detrital modes of sandstones. In: Provenance of Arenites (ed. G.G. Zuffa): 333-361. Reidel, Dordrecht.

Faupl, P., Pober, E., 1991. Zur Bedeutung detritischer Cromspinelle in den Ostalpen: Ophiolitischer Detritus aus der Vardarsutur. In: Jubiläums schrift 20 Jahre Geologische Zusammenarbeit Ősterreich - Ungarn (eds. H. Lobitzer H. and G. Császár), 1: 133-143.

Faupl, P., Wagreich, M., 1992. Cretaceous flysch and pelagic sequences of the Eastern Alps: Correlations, heavy minerals, and palaeogeographical implications. Cretaceous Research, 13: 387-403.

Gawlick, H.-J., Aubrecht, R., Schlagintweit, F., Missoni, S., Plašienka, D., 2015. Ophiolitic detritic material in Kimmeridgian resedimented limestones and its provenance from an eroded obducted ophiolitic nappe stack south of the Northern Calcareous Alps (Austria). Geologica Carpathica, 66: 473-487.

Gross, P., 1963. Stratigrafia a litológia albu v bradlovom pásme medzi Bošácou a Moravským Lieskovým (in Slovak). Geologické práce, Správy, 30: 121-126.

Havlena, V., 1956. Plant spores from Carboniferous pebbles of cannel coal from the Carpathian Cretaceous (in Czech with English summary). Časopis pro mineralogii a geologii, 1: 344-351.

Haško, J., 1977. New geological interpretation of the data about the Havranský vrch and Kozinec klippen near Zázrivá (in Slovak with English abstract). Geologické práce, Správy, 68: 39-47.

Hawthorne, F.C., Oberti, R., Harlow, G.E., Maresch, W.V., Martin, R.F., Schumacher, J.C., Welch, M.D., 2012. Nomenclature of the amphibole supergroup. American Mineralogist, 97: 2031-2048.

Henry, D.J., Guidotti, Ch.W., 1985. Tourmaline as a petrogenetic indicator mineral: an example from the staurolite-grade metapelites of NW Maine. American Mineralogist, 70: 1-15.

Henry, D.J., Novák, M., Hawthorne, F.C., Ertl, A., Dutrow, B.L., Uher, P., Pezzotta, F., 2011. Nomenclature of the tourmaline-supergroup minerals. American Mineralogist, 96: 895-913.

Hók, J., Pešková, I., Potfaj, M., 2009. Lithostratigraphy and tectonic position of the Drietoma Unit (Western part of the Pieniny Klippen Belt, Western Carpathians) (in Slovak with English summary). Mineralia Slovaca, 41: 313-320.

Hubert, J.F., 1962. A zircon-tourmaline-rutile maturity index and the interdependence of the composition of heavy mineral assemblages with the gross composition and texture of sandstones. Journal of Sedimentary Petrology, 32: 440-450.

Ivan, P., Sýkora, M., 1993. Finding of glaucophane-bearing rocks in Cretaceous conglomerates from Jasenov (Križna nappe, Eastern Slovakia) (in Slovak with English summary). Mineralia Slovaca, 25: 29-33.

Ivan, P., Hovorka, D., Méres, Š., 1996. Gabbroid rocks - a newly-found member of leptyno-amphibolite complex of the Western Carpathians. Slovak Geological Magazine, 3-4/96: 199-203.

Ivan, P., Sýkora, M., Demko, R., 2006. Blueschists in the Cretaceous exotic conglomerates of the Klape Unit (Pieniny Klippen Belt, Western Carpathians): their genetic types and implications. Geologia, 32: 47-63.

Jablonský, J., 1978. Contribution to knowledge of the Albian of the Zliechov Group in the Strážovské vrchy Mts. (in Slovak with English summary). In: Paleogeografický vývoj Západných Karpát (eds. J. Vozár, R. Marschalko, M. Mišík and J. Nemčok): 175-187. GÚDŠ, Bratislava. 
Jablonský, J., 1986. Sedimentological study of the Poruba Formation (Albian-Cenomanian) of the Tatric and Zliechov sequences (in Slovak). Unpublished Ph.D. thesis, Comenius University, Bratislava.

Jablonský, J., 1992. Rossfeld Formation in Krížna and Choč nappes, Western Carpathians. Terra Abstracts, 4, Suppl. 2: p. 37

Jablonský, J., Sýkora, M., Aubrecht, R., 2001. Detritic Cr-spinels in Mesozoic sedimentary rocks of the Western Carpathians (overview of the latest knowledge) (in Slovak with English summary). Mineralia Slovaca, 33: 487-498.

Kamenetsky, V.S., Crawford, A.J., Meffre, S., 2001. Factors controlling chemistry of magmatic spinel: an empirical study of associated olivine, $\mathrm{Cr}$-spinel and melt inclusion from primitive rocks. Journal of Petrology, 42: 655-671.

Kamenický, L., Král', J., 1979. Discussion to the problem of development and structure of the crystalline in the wider region of the Klippen Belt of the West Carpathians (in Slovak with English summary). In: Tektonické profily Západných Karpát (ed. M. Mahel'): 59-63. GÚDŠ, Bratislava.

Kamenický, L., Kátlovský, V., Marschalko, R., Medved', J., 1974 Contribution to characterization of acid magmatites of exotic rocks of the Klippen Belt and other tectonic units of the West Carpathians (in Slovak with English summary). Mineralia Slovaca, 6: 311-322

Konzett, J., Krenn, K., Hauzenberger, Ch., Whitehouse, M. Hoinkes., G, 2012. High-pressure tourmaline formation and fluid activity in Fe-Ti-rich eclogites from the Kreuzeck Mountains, Eastern Alps, Austria. Journal of Petrology, 53: 99-125.

Kováč, M., Plašienka, D., Soták, J., Vojtko, R., Oszczypko, N., Less, Gy., Ćosović, V., Fügenschuh, B., Králiková, S., 2016. Paleogene palaeogeography and basin evolution of the Western Carpathians, Northern Pannonian domain and adjoining areas. Global and Planetary Change, 140: 9-27.

Krische, O., Goričan, Š., Gawlick, H.-J., 2014. Erosion of a Jurassic ophiolitic nappe-stack as indicated by exotic components in the Lower Cretaceous Rossfeld Formation of the Northern Calcareous Alps (Austria). Geologica Carpathica, 65: 3-24.

Krivý, M., 1969. Exotische Gerölle magmatischer Gesteine des westlichen Teiles der Klippenzone (in Slovak with German summary). Acta Geoleologica et Geographica Universitatis Comenianae, Geologica, 18: 165-197.

Leake, B.E., Wooley, A.R., Arps, C.E.S., Birch, W.D., Gilbert, M.C., Grice, J.D., Hawthorne, F.C., Kato, A., Kisch, H.J., Krivovichev, V.G., Linthout, K., Laird, J., Mandarino, J., Maresch, W.V., Nickel, E.H., Rock, N.M.S., Schumacher, J.C., Stephenson, N.C.N., Whittaker, E.J.W, Youzhi, G., 1997 Nomenclature of amphiboles: Report of the subcommittee on amphiboles of the International Mineralogical Association Commission on New Minerals and Mineral Names. Mineralogical Magazine, 61: 295-321.

Lenaz, D., Kamenetsky, V.S., Crawford, A.J., Princivalle, F., 2000. Melt inclusion in detrital spinel from the SE Alps (Italy-Slovenia): a new approach to provenance studies of sedimentary basins. Contributions to Mineralogy and Petrology, 139 748-758.

Lenaz, D., Mazzoli, C., Spišiak, J., Princivalle, F., Maritan, L., 2009. Detrital Cr-spinel in the Šambron-Kamenica Zone (Slovakia): evidence for an ocean-spreading zone in the Northern Vardar suture? International Journal of Earth Sciences, 98: 345-355.

Madzin, J., Plašienka, D., Méres, Š., 2019. Provenance of synorogenic deposits of the Upper Cretaceous-Lower Palaeogene Jarmuta-Proč Formation (Pieniny Klippen Belt, Western Carpathians). Geologica Carpathica, 70: 15-34.

Mahel', M., 1978. Manín unit - partial nappe of the Krížna nappe group (in Slovak with English summary). Mineralia Slovaca, 10 289-309.

Mahel', M., 1980. The Peri-klippen zone: its nearer characterization and significance (in Slovak with English summary). Mineralia Slovaca, 12: 193-207.

Mahel', M., 1981. Penninic units in the Western Carpathians from the point of view of global tectonics (in Slovak with English summary). Mineralia Slovaca, 13: 289-306.
Mahel', M., 1989. Pieniny Klippen Belt from the geodynamic model aspect (in Slovak with English summary). Mineralia Slovaca, 21: 99-108.

Mahel', M., 1986. Geologická stavba Československých Karpát 1. Paleoalpínske jednotky (in Slovak). Veda, Bratislava.

Marschall, H., Ludwig, T., Altherr, R., Kalt, A., Tonarini, S., 2006. Syros metasomatic tourmaline: evidence for very high- $\delta 11 \mathrm{~B}$ fluids in subduction zones. Journal of Petrology, 47: 1915-1942.

Marschall, H.R., Altherr, R., Kalt, A., Ludwig, T., 2008. Detrital, metamorphic and metasomatic tourmaline in high-pressure metasediments from Syros (Greece): intra-grain boron isotope patterns determined by secondary-ion mass spectrometry. Contributions to Mineralogy and Petrology, 155: 703-717.

Marschalko, R., 1986. Evolution and geotectonic consequence of the Cretaceous flysch of the Pieniny Klippen Belt (in Slovak with English summary). Veda, Bratislava

Matějka, A., Andrusov, D., 1931. Aperçu de la géologie des Carpathes occidentales de la Slovaquie centrale et les régions avoisinants. Knihovna Státního Geologického Ústavu ČSR, 13A: 19-163.

Mello, J. (ed.), Boorová, D., Buček, S., Filo, I., Fordinál, K. Havrila, M., Iglárová, L., Kubeš, P., Liščák, P., Maglay, J., Marcin, D., Nagy, A., Potfaj, M., Rakús, M., Rapant, S. Remšík, A., Salaj, J., Siráňová, Z., Te ák, Zuberec, J., Zlinská, A., Žecová, K., 2011. Explanations to the geological map of the Middle Váh valley 1:50 000 (in Slovak with English summary). ŠGÚDŠ, Bratislava.

Méres, Š., 2008. Garnets - an important information resource about source area and parental rocks of the siliciclasic sedimentary rocks (in Slovak). In: "Cambelove dni 2008" Conference. Bratislava: Comenius University, Abstract Book (ed. L'. Jurkovič): 37-43.

Michalík, J., 1994. Notes on the paleogeography and paleotectonics of the Western Carpathian area during the Mesozoic. Mitteilungen der Österreichischen Geologischen Gesellschaft, 86: 101-110

Miller, D.P., Marschall, H.R., Schumacher, J.C., 2009 Metasomatic formation and petrology of blueschist-facies hybrid rocks from Syros (Greece): Implications for reactions at the slab-mantle interface. Lithos, 107: 53-67.

Mišík, M., 1978. Some paleogeographic problems of the Pieniny Klippen Belt (in Slovak, with English summary). In: Paleogeographical Evolution of the West Carpathians (eds. J. Vozár, M. Mišik and J. Nemcok): 147-159. Geological Institute of D. Štúr, Bratislava.

Mišík, M., 1996. O sedimentačnom priestore klapskej jednotky (in Slovak). Mineralia Slovaca, 28: 73-75.

Mišík, M., Marschalko, R., 1988. Exotic conglomerates in Flysch sequences: examples from the West Carpathians. Mémoires de la Société géologique de France, Paris, 154: 95-113.

Mišík, M., Sýkora, M., 1981. Der pieninische exotische Rücken, rekonstruiert aus Geröllen karbonátischer Gesteine kretazischer Konglomerate der Klippenzone und der Manín-Einheit (in Slovak with German summary). Západné Karpaty, séria Geológia, 7: 7-111.

Mišík, M., Jablonský, J., Fejdi, P., Sýkora, M., 1980. Chromian and ferrian spinels from Cretaceous sediments of the West Carpathians. Mineralia Slovaca, 12: 209-228.

Mišík, M., Jablonský, J., Mock, R., Sýkora, M., 1981 Konglomerate mit exotischem Material in dem Alb der Zentralen Westkarpaten - paläogeographische und tektonische Interpretation. Acta Geologica et Geographica Universitatis Comenianae, Geologica, 37: 5-55.

Mišík, M., Mock, R., Sýkora, M., 1977. Die Trias der Klippenzone der Karpaten. Geologický zborník SAV, 28: 27-70.

Mišík, M., Sýkora, M., Mock, R., Jablonský, J., 1991. Paleogene Proč Conglomerates of the Klippen Belt in the West Carpathians, material from Neopieninic Exotic Ridge. Acta Geologica et Geographica Universitatis Comenianae, Geologica, 46: 9-101.

Morimoto, N., Fabries, J., Ferguson, A.K., Ginzburg, I.V., Ross, M., Seifert, F.A., Zussman, J., 1988. Nomenclature of pyroxenes. American Mineralogist, 73: 1123-1133. 
Morton, A.C., Hallsworth, C., 2007. Stability of detrital heavy minerals during burial diagenesis. Developments in Sedimentology, 58: $215-245$.

Oszczypko, N., Salata, D., 2005. Provenance analyses of the Late Cretaceous - Palaeocene deposits of the Magura Basin (Polish Western Carpathians) - evidence from a study of the heavy minerals. Acta Geologica Polonica, 55: 237-267.

Pettijohn, F.J., Potter, P.E., Siever, R., 1987. Sand and Sandstone. Springer, New York.

Plašienka, D., 1995. Mesozoic evolution of Tatric units in the Malé Karpaty and Považský Inovec Mts: implications for the position of the Klape and related units in Western Slovakia. Geologica Carpathica, 46: 101-112.

Plašienka, D., 1996. Cryptic ridges or collisional belts (in Slovak with English summary)? Mineralia Slovaca, 28: 75-79.

Plašienka, D., 2018. Continuity and episodicity in the early Alpine tectonic evolution of the Western Carpathians: how large-scale processes are expressed by the orogenic architecture and rock record data. Tectonics, 37: 2029-2079.

Plašienka, D., 2019. Linkage of the Manín and Klape units with the Pieniny Klippen Belt and Central Western Carpathians: balancing the ambiguity. Geologica Carpathica, 70: 35-61.

Plašienka, D., Soták, J., 2015. Evolution of Late Cretaceous-Palaeogene synorogenic basins in the Pieniny Klippen Belt and adjacent zones (Western Carpathians, Slovakia): tectonic controls over a growing orogenic wedge. Annales Societatis Geologorum Poloniae, 85: 273-285.

Pober, E., Faupl, P., 1988. The chemistry of detrital chromian spinels and its implications for the geodynamic evolution of the Eastern Alps. Geologische Rundschau, 77: 671-670.

Pupin, J.-P., 1980. Zircon and granite petrology. Contributions to Mineralogy and Petrology, 73: 207-220.

Pupin, J.-P., Turco, M.J., 1972. Une typologie originale du zircon accessoire. Bulletin de la Société Française de Minéralogie et de Cristallographie, 95: 348-359.

Rakús, M., Hók, J., 2005. The Manín and Klape units: Lithostratigraphy, tectonic classification, paleogeographic position and relationship to Váhicum (in Slovak with English summary). Mineralia Slovaca, 37: 9-26.

Rybár, A., Kantor, J., 1978. Rádiometrické datovanie vybraných útvarov Západných Karpát vo veku ${ }^{40} \mathrm{Ar} /{ }^{40} \mathrm{~K}$ z erupcií upohlavských konglomerátov Pieninského klippenského pásu (in Slovak). Unpublished manuscript, Geofond archive, Bratislava.

Straka, A., 2011. Geochemistry of flysch succession sediments from selected localities of Magura Unit and Pieniny Klippen Belt (in Slovak with English summary). MSc. thesis, Comenius University, Bratislava.

Stern, G., Wagreich,M., 2013. Provenance of the Upper Cretaceous to Eocene Gosau Group around and beneath the Vienna Basin (Austria and Slovakia). Swiss Journal of Geosciences, 106: 505-527.

Sýkora, M., Halásová, E., Boorová, D., 1997. Blue amphiboles and microfossils from the Mesozoic basement of the Vienna Basin (borehole Smolinské 27), Slovakia. Mineralia Slovaca, 29: 227-233.

Šilar, J., 1956. Výskyt karbonského uhlí v senónských zlepencích v Považí u Púchova (in Czech). Časopis pro mineralogii a geologii, 1: 334-343.

Šímová, M., 1982. Eclogitoid rock in pebbles of Cretaceous conglomerates of Klippen Belt (in Slovak with English summary). Geologické práce, Správy, 77 : 55-74.

Šímová, M., 1985a. Petrochemische Kriterien der Genese und strukturellen Zuordnung von Magmatiten aus exotischen Geröllen kretazischer Konglomerate der Klippenzone der Westkarpaten. Acta Geologica et Geographica Universitatis Comenianae, Geologica, 39: 85-99.

Šímová, M., 1985b. Granitoide Gesteine der Gerölle mittelkretazischer Konglomerate der Klippenzone der Westkarpaten. Acta Geologica et Geographica Universitatis Comenianae, Geologica, 40: 79-88.

Šímová, M., 1985c. Magmatogene Gesteine kretazischer Konglomerate des westlichen Teiles der Klippen- und Manín-
Zone der Westkarpaten (in Slovak with German summary). Západné Karpaty, séria Mineralógia, petrografia, geochémia, metalogenéza, 10: 9-110.

Šímová, M., Šamajová, E., 1982. Lawsonite from rock pebbles in the Cretaceous conglomerate of the Pieniny Klippen Belt (in Slovak with English summary). Mineralia Slovaca, 14: 431-441.

Tomaś, A., Olszewska, B., Golonka, J., Cieszkowski, M., Krobicki, M., 2004. Devonian exotics in the Pieniny Klippen Belt flysch and their significance for the Paleozoic plate tectonic reconstruction of the West Carpathians. Geolines, 17: 93-94.

Túnyi, I., Márton, E., 2002. Cenozoic paleomagnetic rotations in the Inner Western Carpathians. Geologica Carpathica, 53 (spec. iss.): $128-129$.

Uher, P., Broska, I., 1996. Post-orogenic Permian granitic rocks in the Western Carpathian-Pannonian area: geochemistry, mineralogy and evolution. Geologica Carpathica, 47: 311-321.

Uher, P., Marschalko, R., 1993. Typology, zoning and chemistry of zircon from main types of granitic and rhyolitic pebbles in conglomerates of the Pieniny Klippen Belt Cretaceous flysch (Western Slovak segment, Western Carpathians). Geologica Carpathica, 44: 113-121.

Uher, P., Pushkarev, Yu., 1994. Granitic pebbles of the Cretaceous flysch of the Pieniny Klippen Belt, Western Carpathians: U/Pb zircon ages. Geologica Carpathica, 45: 375-378.

Uher, P., Marschalko, R., Martini, E., Puškelová, L'., Streško, V., Toman, B., Walzel, E., 1994. Geochemical characterization of granitic rocks pebbles from Cretaceous-Paleogene flysch of the Pieniny Klippen Belt. Geologica Carpathica, 44: 113-122.

Von Eynatten, H., Gaupp, R., 1999. Provenance of Cretaceous synorogenic sandstones in the Eastern Alps: constraints from framework petrography, heavy mineral analysis and mineral chemistry. Sedimentary Geology, 124: 81-111.

Whitney, D.L., Evans, B.W., 2010. Abbreviations for names of rock-forming minerals. American Mineralogist, 95: 185-187.

Wagreich, M., 2003. A slope-apron succession filling a piggyback basin: the Tannheim and Losenstein Formations (AptianCenomanian) of the eastern part of the Northern Calcareous Alps (Austria). Mitteilungen der Österreichischen Geologischen Gesellschaft, 93: 31-54.

Wagreich, M., Faupl, P., Schlagintweit, F., 1995. Heavy minerals from Urgonian Limestone pebbles of the Northern Calcareous Alps (Austria, Bavaria): further evidence for an intra-Austroalpine suture zone. Geologica Carpathica, 46: 197-204.

Winkler, W., 1988. Mid- to early Late Cretaceous flysch and melange formations in the western rart of the Eastern Alps. Paleotectonic Implications. Jahrbuch der Geologischen Bundesanstalt, 131: 341-389.

Winkler, W., Bernoulli, D., 1986. Detrital high-pressure/low temperature minerals in a late Turonian flysch sequence of the eastern Alps (western Austria): Implications for early Alpine tectonics. Geology, 14: 598-601.

Winkler, W., Ślączka, A., 1992. Sediment dispersal and provenance in the Silesian, Dukla and Magura flysch nappes (Outer Carpathians, Poland). Geologische Rundschau, 81: 371-382.

Winkler, W., Ślączka, A., 1994. A Late Cretaceous to Paleogene geodynamic model for the Western Carpathians in Poland. Geologica Carpathica, 45: 71-82.

Woletz, G., 1963. Charakteristische Abfolgen der Schwermineralgehalte in Kreide- und Alttertiär-Schichten der nördlichen Ostalpen. Jahrbuch der Geologischen Bundesanstalt, 106: 89-119.

Zat'ko, F., Sýkora M., 2006. Pebbles of siliceous clastics and siliceous rocks in conglomerates of flysch sequences (Albian, Cenomanian) in vicinity of the Považská Bystrica Town, Klape Unit, Pieniny Klippen Belt, Western Carpathians. Geologia, 32: $1,65-77$.

Zoubek, V. 1931. Caractéristique de quelques roches cristallophylliennes et éruptives des galęts exotiques des conglomérats sénoniens et paléogčnes des Carpathes occidentales. Knihovna Státního Geologického Ústavu ČSR, 13: 353-358. 